\title{
Involvement of TRPC Channels in CCL2-Mediated Neuroprotection against Tat Toxicity
}

\author{
Honghong Yao, ${ }^{1}$ Fuwang Peng, ${ }^{1}$ Navneet Dhillon, ${ }^{1}$ Shannon Callen, ${ }^{1}$ Sirosh Bokhari, ${ }^{1}$ Lisa Stehno-Bittel, ${ }^{1,2}$ \\ S. Omar Ahmad, ${ }^{3}$ John Q. Wang, ${ }^{4}$ and Shilpa Buch ${ }^{1}$ \\ Departments of ${ }^{1}$ Molecular and Integrative Physiology, ${ }^{2}$ Physical Therapy and Rehabilitation Science, and ${ }^{3}$ Occupational Therapy and Therapeutic Science, \\ University of Kansas Medical Center, Kansas City, Kansas 66160, and ${ }^{4}$ Department of Basic Medical Science, University of Missouri-Kansas City School of \\ Medicine, Kansas City, Missouri 64108
}

Chemokine (C-C motif) ligand 2 (CCL2), also known as monocyte chemoattractant protein-1, plays a critical role in leukocyte recruitment and activation. In the present study, we identify an additional role for CCL2 that of neuroprotection against HIV-1 transactivator protein (Tat) toxicity in rat primary midbrain neurons. Furthermore, we report the involvement of transient receptor potential canonical (TRPC) channels in CCL2-mediated neuroprotection. TRPC are $\mathrm{Ca}^{2+}$-permeable, nonselective cation channels with a variety of physiological

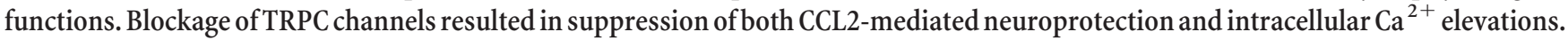
Parallel but distinct extracellular signal-regulated kinase (ERK)/cAMP response element-binding protein (CREB) and Akt/nuclear factor $\kappa \mathrm{B}(\mathrm{NF}-\kappa \mathrm{B})$ pathways were involved in the CCL2-mediated neuroprotection. Blocking TRPC channels and specific downregulation of TRPC channels 1 and 5 resulted in suppression of CCL2-induced ERK/CREB activation but not Akt/NF- $\kappa$ B activation. In vivo relevance of these findings was further corroborated in wild-type and CCR2 knock-out mice. In the wild-type but not CCR2 knock-out mice, exogenous CCL2 exerted neuroprotection against intrastriatal injection of HIV-1 Tat. These findings clearly demonstrate a novel role of TRPC channels in the protection of neurons against Tat through the CCL2/CCR2 axis.

Key words: CCL2; TRPC; midbrain neurons; $\mathrm{Ca}^{2+}$; ERK; Akt

\section{Introduction}

Extensive neuronal damage and loss is a hallmark of HIVassociated dementia (HAD), a term used to describe the range of cognitive and motor impairments that occur in $10-20 \%$ of individuals suffering from AIDS (Bachis et al., 2003). There is no conclusive evidence of direct neuronal infection by HIV-1, and neuronal death is considered to be a consequence of the toxic effects of viral and cellular neurotoxins that are released from virus-infected and/or activated cells (Eugenin et al., 2003). One of the potent viral toxins implicated in neuronal death is the transactivator protein (Tat). Tat can be secreted from infected cells and can be taken up by neighboring non-infected cells, including neurons (Liu et al., 2000). Indeed, Tat causes cell death in various neuronal populations in vitro, including midbrain dopaminergic neurons (Aksenova et al., 2006) and striatal neurons (Singh et al.,

\footnotetext{
Received June 18, 2008; revised 0ct. 31, 2008; accepted Dec. 24, 2008.

This work was supported by National Institutes of Health Grants MH62969, RR016443, MH-068212, DA020392, and DA024442 (S.B.) and a mentored fellowship from the Parker B. Francis Foundation (N.D.). We thank Dr. K. Walsh (Tufts University School of Medicine, Medford, MA) for providing adenoviral constructs for the WT and dominantinterfering forms of Akt. We also thank Dr. S. Maggirwar (University of Rochester Medical Center, Rochester, NY) for providing us with recombinant adenovirus vectors coexpressing either GFP and full-length RelA or mutant RelA. We thank Dr. Y. Jia (The Jackson Laboratory) for technical support with siRNA TRPC.

Correspondence should be addressed to Dr. Shilpa Buch, Department of Molecular and Integrative Physiology, 5000 Wahl Hall East, University of Kansas Medical Center, 3901 Rainbow Boulevard, Kansas City, KS 66160. E-mail: sbuch@kumc.edu.

D0I:10.1523/JNEUROSCI.2781-08.2009

Copyright $\odot 2009$ Society for Neuroscience $\quad$ 0270-6474/09/291657-13\$15.00/0
}

2004). Neurotoxic effects of Tat have also been demonstrated in vivo in murine models (Zauli et al., 2000).

In the brains of individuals with HAD, upregulation of chemokines in the CNS is often considered a correlate of neuroinflammation. However, recent increasing evidence raises the possibility that, in addition to their role as chemoattractants, chemokines might also act as neurotransmitters or neuromodulators (Rostène et al., 2007). A classic example of this is the chemokine fractalkine, which is not only a neuroimmune modulator recruiting peripheral macrophages into the brain but can also function as a neuroprotective factor (Tong et al., 2000). CCL2, a member of the $\mathrm{C}-\mathrm{C}$ subfamily of chemokines, has been shown to be the most potent of a variety of monocyte chemoattractants with diverse functions. Similar to fractalkine, CCL2 has also been demonstrated to protect cardiac myocytes from hypoxia-induced apoptosis (Tarzami et al., 2005) and fetal neuronal cultures against HIV-1 Tat toxicity (Eugenin et al., 2003). However, whether CCL2 can protect midbrain neurons, the target for HIV-1/HIV proteins, remains unexplored.

Recent studies have revealed the role of transient receptor potential canonical (TRPC) channels, $\mathrm{Ca}^{2+}$-permeable, nonselective cation channels, in neuroprotection (Jia et al., 2007). Transient receptor potential (TRP) channels are formed by homomeric or heteromeric complexes of TRP proteins that constitute at least three subfamilies: TRPC, TRP vanilloid (TRPV), and TRP melastatin (Montell et al., 2002; Clapham, 2003). These channels play critical roles in diverse physiological processes 
ranging from sensation to male fertility. Mammalian TRPC channels can be activated by G-protein-coupled receptors such as CCR2 (Clapham, 2003; Ramsey et al., 2006; Ambudkar and Ong, 2007). Because CCL2/CCR2 axis is known to stimulate phospholipase C- $\beta$ (PLC- $\beta$ ) to subsequently activate TRPV in dorsal root ganglia neurons (Jung et al., 2008), we rationalized that CCL2mediated neuroprotection involved TRPC channels.

In the present study, we show direct evidence that CCL2 signaling in the midbrain neurons may contribute to the maintenance of neuronal survival and reveal a previously unknown role of TRPC in CCL2-mediated neuroprotection.

\section{Materials and Methods}

Reagents. Recombinant CCL2 was purchased from R \& D Systems, and Tat1-72 was obtained from the AIDS Research and Reference Reagent Program of the National Institutes of Health. The specific phosphatidylinositol-3' kinase (PI3K) inhibitor LY294002 [2-(4morpholinyl)-8-phenyl-1(4H)-benzopyran-4-one], the PLC inhibitor U73122 (1-[6[[(17 $\beta)$-3-methoxyestra-1,3,5(10)-trien-17-yl]amino]hexyl]-1H-pyrrole-2,5-dione), and MEK1/2 inhibitor U0126 [1,4diamino-2,3-dicyano-1,4-bis(o-aminophenylmercapto)butadiene] were purchased from Calbiochem. CCR2 antagonist RS102895 (1'-[2[4-(trifluoromethyl)phenyl] ethyl]-spiro[4H-3,1-benzoxazine-4,4' peperidin]-2(1H)-one, TRPC blocker SKF96365 [1-2-(4-methoxyphenyl)-2-[3-(4-methoxyphenyl)propoxy] ethyl-1 $H$-imidazole], DAG analog 1-oleoyl-2-acetyl-sn-glycerol (OAG), and nuclear factor- $\kappa \mathrm{B}$ (NF$\kappa \mathrm{B})$ inhibitor $\mathrm{N}$-a-tosyl-L-phenyl alanine chloromethyl ketone (TPCK) were purchased from Sigma. 2-Aminoethoxydiphenyl borate (2-ApB) was obtained from Tocris Cookson. Anti-TRPC1, TRPC5, andTRPC6 antibodies were purchased from Alomone Labs.

Animals. Pregnant female Sprague Dawley rats and C57BL/6N wildtype (WT) mice were purchased from (Charles River Laboratories). CCR2 knock-out (KO) mice (Taconic Farms) have been backcrossed 10 generations to a $\mathrm{C} 57 \mathrm{BL} / 6 \mathrm{~N}$ inbred background. All animals were housed under conditions of constant temperature and humidity on a $12 \mathrm{~h}$ light/ dark cycle, with lights on at 7:00 A.M. Food and water were available ad libitum. All animal procedures were performed according to the protocols approved by the Institutional Animal Care and Use Committee of the University of Kansas Medical Center.

Primary neuronal cultures. Primary rat midbrain neurons were prepared as described previously with slight modifications (Yao et al., 2005). Briefly, midbrain tissues were dissected from embryonic day 13-14 Sprague Dawley rats and dissociated with mild mechanical trituration. Dissociated cells were seeded firstly at $5 \times 10^{5}$ cells per well on 24 -well culture plates and maintained in DMEM/F-12 supplemented with 10\% fetal bovine serum and $1 \%$ antibiotic. After $1 \mathrm{~d}$, the cultures were supplemented with serum-free $\mathrm{Neu}-$ robasal medium containing B27 (50:1), 2 mm glutamax, and 1\% antibiotic. After $7 \mathrm{~d}$, the initial plating cultures consisted of $90 \%$ neuron-specific nuclear protein-immunoreactive (IR) neurons of which $3 \%$ were tyrosine hydroxylase $(\mathrm{TH})$-positive neurons. Similar procedure was used to isolate midbrain neurons from WT and CCR2 KO mice.

Surgery and microinjection. Ten-week-old C57BL/6N and CCR2 KO mice ( $n=3$ per group) were anesthetized with $2.5 \%$ isoflurane and placed in a stereotaxic apparatus for cannula implantation. A permanent 26-gauge stainless steel guide cannula (C315G; Plastics One) was implanted into the right striatum using the following stereotaxic coordinates: anteroposterior, $+0.86 \mathrm{~mm}$ posterior; mediolateral, $-1.8 \mathrm{~mm}$ lateral to midline; and dorsoventral, $-3.5 \mathrm{~mm}$ to bregma according to Paxinos and Franklin (2001) mouse brain atlas. The guide cannula was secured in place using gel adhesive and dental cement applied sequentially to the skull. A 33-gauge stainless steel dummy cannula was used to seal the guide cannula when not in use. After surgery, the animals were housed individually to avoid damage to guide and dummy cannulas.

After striatum cannulation, animals were allowed to recover for $7 \mathrm{~d}$. For the CCL2+Tat group, $2 \mu \mathrm{l}$ of CCL2 $(400 \mathrm{ng} / \mu \mathrm{l})$ was microinjected into the striatum once daily for $2 \mathrm{~d}$, followed by microinjection of Tat $(500 \mathrm{ng} / \mu \mathrm{l})$ for another $2 \mathrm{~d}$. The control and Tat alone groups of mice were injected with the same volume of Tat or sterile saline. All microin-
Table 1. siRNA sequence of rat TRPC1, TRPC5, and TRPC6

\begin{tabular}{lll}
\hline Items & Direction & Sequence \\
\hline TRPC1 siRNA-1 & Sense & CCAUAACUAUUGAAAACGAtt \\
& Antisense & UCGUUUUCAAUAGUUAUGGta \\
TRPC1 siRNA-2 & Sense & GAUCUGUCAAAAUUCCGAAtt \\
& Antisense & UUCGGAAUUUUGACAGAUCtt \\
TRPC1 siRNA-3 & Sense & GUUUCGUCUUGAUAUCUAUtt \\
& Antisense & AUAGAUAUCAAGACGAAACt \\
TRPC5 siRNA-1 & Sense & GAGUGGAAGUUGGGAGAAtt \\
& Antisense & UUCUCGCAAACUUCCACUCaa \\
TRPC5 siRNA-2 & Sense & GCCUGAUCCAAAAUCAACAtt \\
& Antisense & UGUUGAUUUUGGAUCAGGCtg \\
TRPC5 siRNA-3 & Sense & CACUCUUCGCGAUAUGAAtt \\
& Antisense & UUCGAUAUCGCGAGAGUGct \\
TRPC6 siRNA-1 & Sense & GCAUCAUCGAUGCAAAUGAtt \\
& Antisense & UCAUUUGCAUCGAUGAUGCtc \\
TRPC6 siRNA-2 & Sense & GUUUCUCCAGAAUAGCUUAtt \\
& Antisense & UAAGCUAUUCUGGAGAAACtt \\
TRPC6 siRNA-3 & Sense & CAUACAUGUUUAAUGAUCAtt \\
& Antisense & UGAUCAUUAAACAUGUAUGct \\
\hline
\end{tabular}

jections were performed using a 33-gauge stainless steel injector connected to a $10 \mu \mathrm{l}$ syringe, which was operated by an infusion pump set at the rate of $0.4 \mu \mathrm{l} / \mathrm{min}$. An additional minute was allowed for diffusion and prevention of backflow through the needle track before the injector was withdrawn. Histological verification of the striatum cannula was performed at the end of each experiment.

MTT assay. Cell viability was measured by mitochondrial dehydrogenases [3(4,5-dimethylthiazol-2-yl)-2.5y diphenyltetrazolium bromide (MTT) (Sigma)] assay as described previously (Yao et al., 2005).

Annexin-V staining using flow cytometry. Neuronal apoptosis was estimated using the Annexin-V fluorescein (FITC) apoptosis detection kit (Invitrogen) according to the instructions of the manufacturer as described previously (Yao et al., 2005).

Hoechst staining. To quantify apoptotic neurons, cells were fixed and stained with $5 \mu \mathrm{M}$ Hoechst 33324 (Invitrogen) for $15 \mathrm{~min}$ at room temperature. The morphological features of apoptosis (cell shrinkage, chromatin condensation, and fragmentation) were monitored by fluorescence microscopy (Nikon TE2000E microscope).

Terminal deoxynucleotidyl transferase-mediated biotinylated UTP nick end labeling staining. Primary neurons treated with Tat and/or CCL2 were fixed for $30 \mathrm{~min}$ with $4 \%$ paraformaldehyde at room temperature. Fixed cells were then permeabilized with $1 \%$ Triton X-100 for $30 \mathrm{~min}$, followed by staining with terminal deoxynucleotidyl transferasemediated biotinylated UTP nick end labeling (TUNEL) reaction mixture for $60 \mathrm{~min}$, according to the instructions of the manufacturer (Roche) (Peng et al., 2008).

Analyses of neuronal dendrites. Primary rat midbrain neurons were stained with anti-microtubule-associated protein-2 (MAP-2) antibody, and dopaminergic neurons were identified with the anti-TH as described previously with minor modifications (Zhou et al., 2006). For the measurement of the dendrite length, images of individual MAP-2/THpositive neurons were recorded. The length of each fiber originating from each neuronal cell body and subsequent branches was measured, and a sum of total dendrite length for each neuron was calculated using Image Pro-plus software. For each well, 50 neurons were analyzed for total dendrite length. Results were expressed as a percentage of the control cultures using a Nikon TE2000E microscope with a digital camera (Photometrics) (Zhou et al., 2006).

Reverse transcription-PCR. Total RNA was extracted with TRI reagent as detailed by the instructions from manufacturer. Nest PCR was used to detect CCR2 mRNA, the reaction mixture was prepared according the instructions of the Qiagen OneStep RT-PCR kit and the Verso 1-step RT-PCR kit (Thermo Fisher Scientific). The primers for CCR2 (GenBank accession number U77349) used in the first round were as follows: upstream primer, 5'-CGCAGAGTTGACAAGTTGTG-3'; and downstream primer, 5'AATACCAGGGATAGAGTG-3'. The primers used in the second round 
A

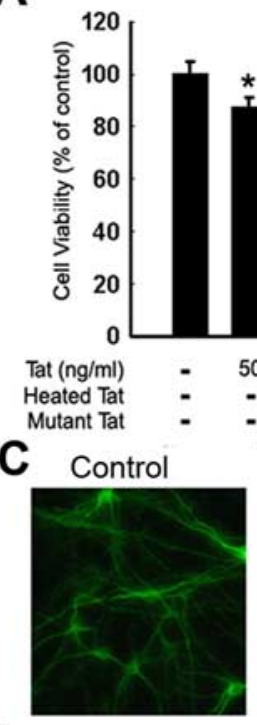

E

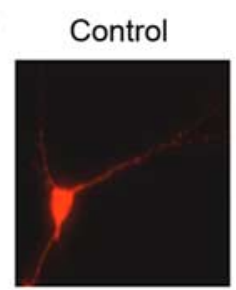

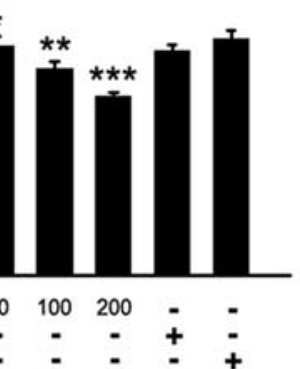

Tat

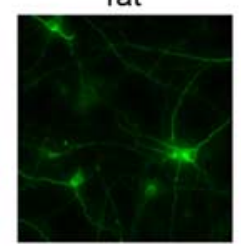

Tat

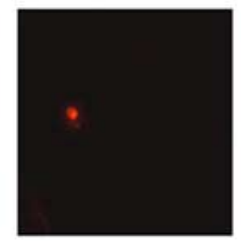

H
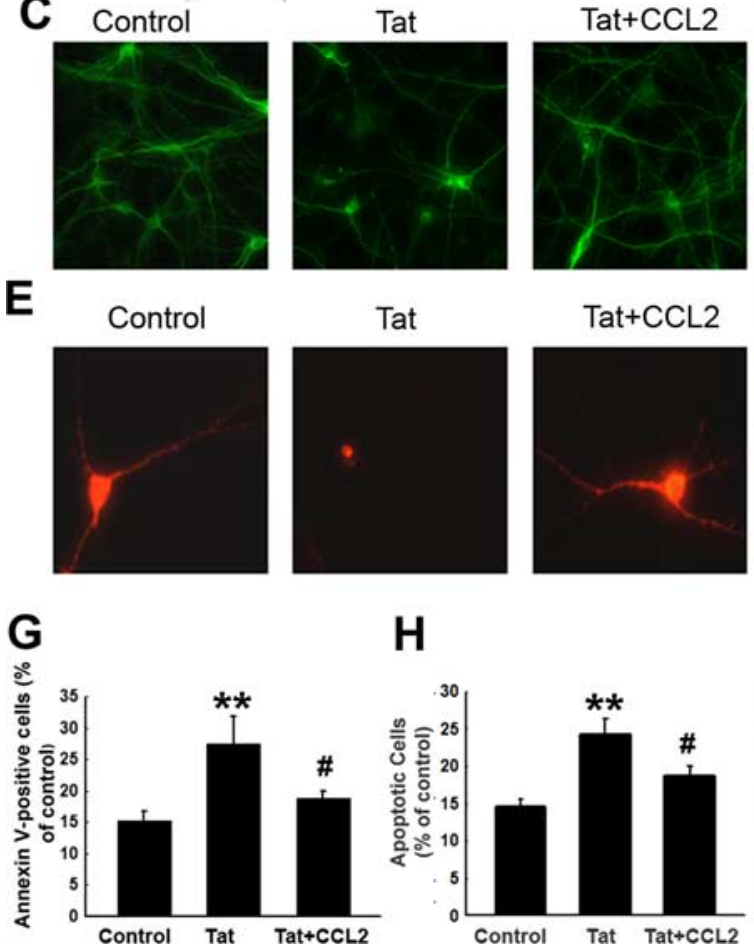

Tat+CCL2
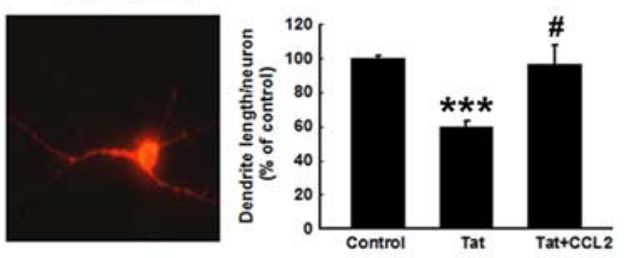

I

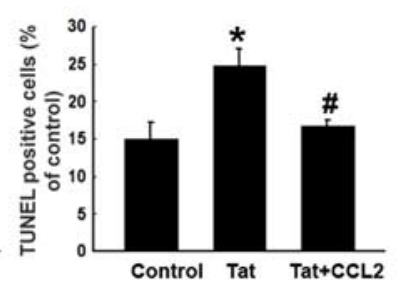

Figure 1. CCL2 exerts neuroprotection against Tat toxicity. $A$, Effects of varying concentrations of Tat on the cell survival of rat primary midbrain neurons using the MTT assay. B, CCL2 protects rat primary midbrain neurons against Tat neurotoxicity. $\boldsymbol{C}$, Immunostaining of rat primary midbrain neurons treated with Tat and/or CCL2 for $24 \mathrm{~h}$ with anti-MAP-2 antibody. D, Densitometric scan of neuritis (from $C$ ) expressed as a ratio of neurite length/neuron. $E$, Immunostaining of rat primary midbrain neurons treated with Tat and/or CCL2 using anti-TH antibody. $\boldsymbol{F}$, Densitometric scan of neuritis (from $\boldsymbol{E}$ ) using the same method as in $\boldsymbol{D}$. Rat primary midbrain neurons treated with Tat and/or CCL2 were stained for the apoptosis marker Annexin V-FITC using flow cytometric analysis $(\boldsymbol{G})$, Hoechst 33342 staining monitored by fluorescence microscopy $(\boldsymbol{H})$, and TUNEL staining $(\boldsymbol{I})$. Quantification of Annexin V-positive $(\boldsymbol{G})$, Hoechst-positive $(\boldsymbol{H})$, and TUNEL-positive $(\boldsymbol{I})$ cells as the percentage of total cells. The data are presented as mean \pm SEM of four individual experiments. All the data in these figures are presented as mean \pm SEM of four individual experiments. ${ }^{*} p<0.05 ;{ }^{* *} p<0.01{ }^{* * *} p<0.001$ versus control group; ${ }^{\#} p<0.05$; ${ }^{\# \#} p<0.001$ versus Tat $(200 \mathrm{ng} / \mathrm{ml}$ ) group.

were as follows: upstream primer, 5'-AGACAGGGATGCTGCTTA-3'; and downstream primer, 5'-AATACCAGGGATAGAGTG-3'.

For TRPC, the primer pair sequences for TRPC2, TRPC3, TRPC4, TRPC5, and TRPC7 were the same as those published by Wang et al. (2004). Primers for TRPC1 and TRPC6 are as follows: (1) TRPC1 (GenBank accession number NM011643): upstream primer, 5'-CTTGACAAACGAGGACTACTA-3'; and downstream primer, 5' -TTCCAACCCTTCATACCA-3'; (2) TRPC6 (GenBank accession number AB051214): upstream primer, 5'-GTCCATTCATGAAGTTCGTAGCAC-3'; and downstream primer, 5'-AATATTCTTTGGGGCCTTGAGTCC- $3^{\prime}$. The mixtures were annealed at $50^{\circ} \mathrm{C}(1 \mathrm{~min})$, extended at $72^{\circ} \mathrm{C}(2 \mathrm{~min})$, and denatured at $94^{\circ} \mathrm{C}(1 \mathrm{~min})$ for 35 cycles. The PCR product was visualized by electrophoresis on $1.2 \%$ agarose gels.

Measurement of calcium transients. The changes in intracellular $\mathrm{Ca}^{2+}$ $\left(\left[\mathrm{Ca}^{2+}\right]_{\mathrm{i}}\right)$ were monitored using Fluo-4 AM dissolved in dimethylsulf- oxide. The rat midbrain neurons in $35 \mathrm{~mm}$ culture dishes were rinsed twice with physiological solution (PS) (in mM: $140 \mathrm{NaCl}, 5 \mathrm{KCl}, 1 \mathrm{CaCl}_{2}$, $0.5 \mathrm{MgCl}_{2}, 10$ glucose, and 5.5 HEPES, pH 7.4), followed by incubation in PS containing $5 \mu \mathrm{M}$ Fluo-4 AM (Invitrogen) at $37^{\circ} \mathrm{C}$ for $40 \mathrm{~min}$. Cells were then rinsed twice with the PS, mounted on a perfusion chamber, and scanned every second using confocal microscopy (Fluoview 300; Olympus). Fluorescence excitation at $488 \mathrm{~nm}$ was provided by an argon laser, and the emitted light $(515 \mathrm{~nm})$ captured along with transmitted images. All analyses of $\left[\mathrm{Ca}^{2+}\right]_{i}$ were processed at a single-cell level and are expressed as the relative fluorescence intensity. For ratiometric analysis, cells were loaded (using procedures described above) with Fluo-4 and Fura Red. Fluorescence values from subcellular regions were calculated as mean \pm SE pixel values, and a ratio was calculated by using the Fluo-4/Fura Red combination. All of these studies were done at least four times.

Western blotting. Treated cells were lysed using the Mammalian Cell Lysis kit (Sigma) and the NE-PER Nuclear and Cytoplasmic Extraction kit (Pierce). Western blots were then probed with antibodies recognizing the phosphorylated forms of extracellular signalregulated kinase (ERK) (1:500; Cell Signaling Technology), Akt (1:500; Cell Signaling Technology), NF- $\kappa$ B p65 (1:1000; Abcam), and cAMP response element-binding protein (CREB) (1:1000; Cell Signaling Technology). Secondary antibodies were alkaline phosphatase conjugated to goat anti-mouse/rabbit IgG (1:5000). Signals were detected by chemiluminescence (Pierce). All of the Western blot experiments were repeated three times individually, and representative blots are presented in the figures.

Adenovirus infection. Primary midbrain neurons were infected with adenoviral constructs containing the WT or dominant-interfering forms of Akt (kind gift from Dr. K. Walsh, Tufts University School of Medicine, Medford, MA). In addition, neurons were also infected with recombinant adenovirus vectors coexpressing both green fluorescent protein (GFP) and fulllength RelA or GFP and a transcriptionally inert RelA mutant (RelA1-300) (both vectors were a kind gift from Dr. S. Maggirwar, University of Rochester Medical Center, Rochester, NY) used at a multiplicity of infection of 50 as described previously (Fujio and Walsh, 1999; Kaul et al., 2007). Neurons infected for $48 \mathrm{~h}$ with adenovirus constructs were subsequently treated with Tat and/or CCL2, followed by assessment of cell survival as described above.

Short interfering RNA transfection. Short interfering RNAs (siRNAs) targeted against TRPC1, TRPC5, and TRPC6 were obtained from Ambion. The sequences of these siRNAs are listed in Table 1. For siRNA transfection, we used the rat Neuron Nucleofector kit (Amaxa) according to the instructions of the manufacturer. Briefly, dissociated cells were resuspended in transfection medium, mixed with the respective siRNAs (200 nM), and electroporated using the fixed program (O-03) for optimal neuronal transfection. Cells were then quickly centrifuged, resuspended, and plated. The knockdown efficiency of siRNAs was determined after $7 \mathrm{~d}$ of transfection by Western blotting.

Immunohistochemistry. Mice were perfused by transcardial perfusion using chilled $4 \%$ paraformaldehyde. Free-floating sections encompass- 
ing the entire midbrain were sectioned at $40 \mu \mathrm{m}$ on a cryostat. For TH immunostaining, tissue sections were incubated with primary antibodies overnight at $4^{\circ} \mathrm{C}$. Primary antibodies used in this study were as follows: rabbit anti-TH (1: 4000; Sigma). Immunostaining was visualized by using $3,3^{\prime}$-diaminobenzidine as the substrate.

Stereology. Quantitative (neuronal number) estimates of TH-positive cell bodies were performed ipsilaterally in the areas of interest. This brain area was defined anatomically by atlas and the agreement of three neuroscientists. Random sampling was ensured by selecting every third section of the substantia nigra pars compacta $(\mathrm{SNc})$, from a random initial sort. Counts used the optical fractionator method and the Stereologer software package. The microscope used was a Nikon Eclipse 80i, linked to a Sony 3CCD Color Digital Video Camera, operating an Advanced Scientific Instrumentation MS-2000 motorized stage input into a Dell Precision 650 Server and a high-resolution plasma monitor. The area of interest was defined with $4 \times / 1.3$ aperture dry lenses, and the stereology was performed at high magnification with $100 \times / 1.4$ aperture oil immersion lenses (yielding $3600 \times$ ). This allowed for clear visualization of the nucleolus and precise definition of the cell walls. When the areas of interest were identified, the area was precisely outlined and checked against an atlas. The inclusion grid was randomly applied by the software, and highresolution microscopy was used to count $\mathrm{TH}$ positive neurons.

Descriptive data was presented for numbers of TH-positive neurons per animal. Independent samples tests ( $t$ test for equality of means) are presented between groups and within groups for cell counts of TH-positive cells. Univariate ANOVA was performed for cell counts.

Statistical analysis. Data were expressed as mean \pm SEM. Significance of differences between control and samples treated with various drugs was determined by one-way ANOVA, followed by post hoc least significant difference test. Values of $p<0.05$ were considered as statistically significant.

\section{Results}

\section{CCL2 attenuated Tat cytotoxicity in rat primary}

\section{midbrain neurons}

To assess the toxic effects of Tat, rat primary midbrain neurons were exposed to varying concentrations of Tat protein for $24 \mathrm{~h}$, and cell viability was assessed by MTT assay. As shown in Figure $1 A$, there was a concentration-dependent effect of Tat on cell viability in rat primary neurons exposed to Tat. Increasing concentrations of Tat $(50,100$, and $200 \mathrm{ng} / \mathrm{ml})$ resulted in decreasing cell viability by $13 \%(p<0.05), 21 \%(p<0.05)$, and $32 \%(p<$ $0.001)$, respectively. Treatment of neuronal cells with heatinactivated or mutant Tat, however, did not exert cytotoxicity in these cells. These findings were also confirmed in a neuronal cell line, SH-SY5Y (data not shown).

To test whether CCL2 could abrogate Tat cytotoxicity, rat neurons were preincubated with varying concentrations of CCL2 (50, 100, and $200 \mathrm{ng} / \mathrm{ml}$ or 5.5, 11, and $22 \mathrm{nM}$, respectively) for 30 $\mathrm{min}$, followed by the exposure of cells to Tat $(200 \mathrm{ng} / \mathrm{ml})$. Twenty-four hours later, cell viability was measured using the MTT assay. As shown in Figure $1 B$, pretreatment of cells with CCL2 resulted in a concentration-dependent increase in cell via-
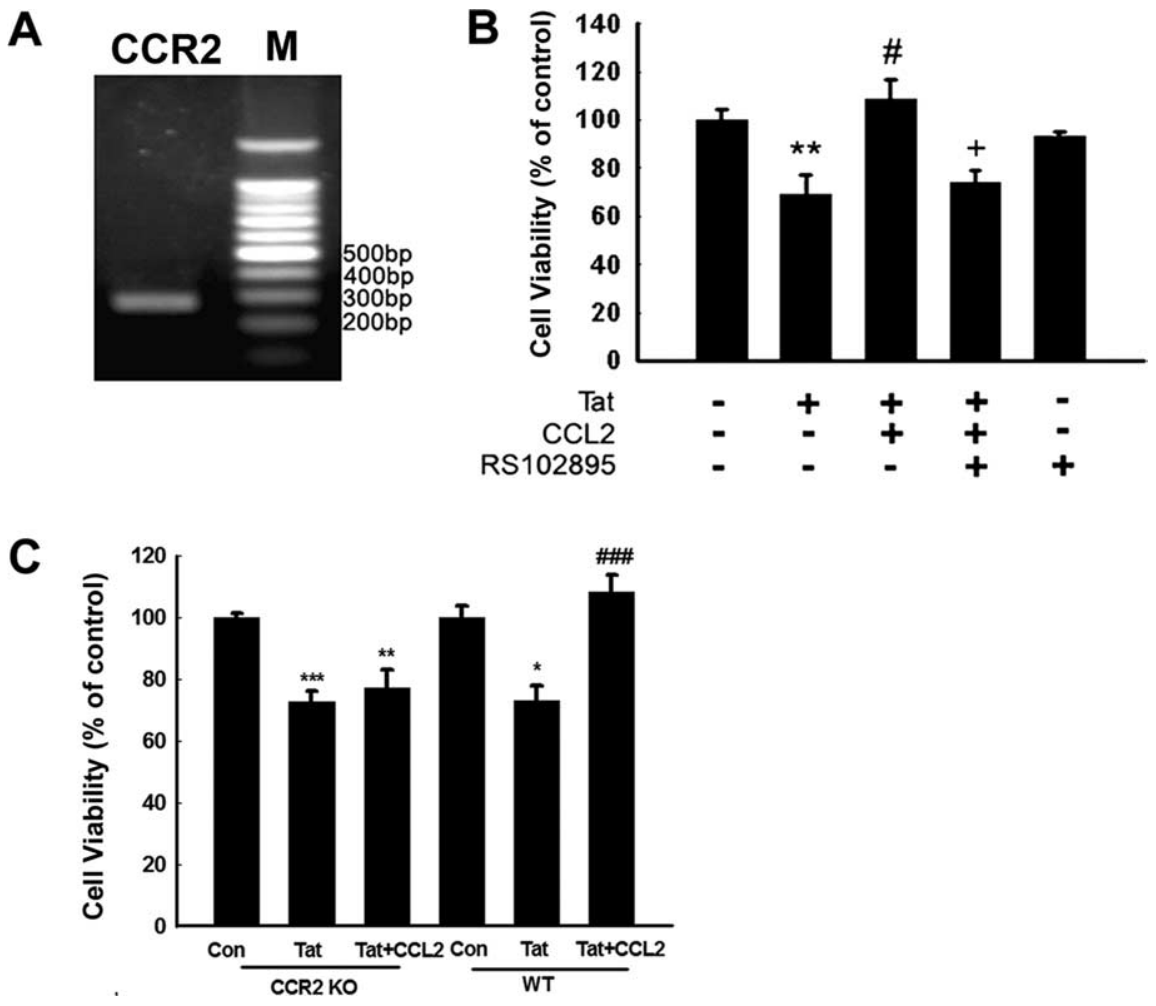

Figure 2. $\quad C C R 2$ is critical for CCL2-mediated neuroprotection. $A, R T-P C R$ of CCR2 RNA in rat primary midbrain neurons; $M$ A marker. $\boldsymbol{B}, \mathrm{RS} 102895$ abolished CCL2-mediated neuroprotection. The data are presented as mean \pm SEM of four for cell viability using MTT assays. The data are presented as mean \pm SEM of four individual experiments. ${ }^{*} p<0.05,{ }^{* *} p<0.01$, ${ }_{* * *} p<0.001$ versus control group. ${ }^{\# \#} p<0.001$ versus Tat-treated group.

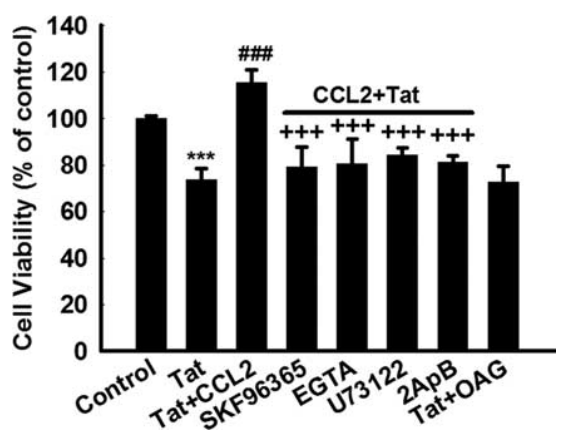

Figure 3. TRPC channels are required for CCL2-mediated neuroprotection. Cell viability of rat primary neurons exposed to Tat and/or CCL2 in the absence or presence of indicated drugs (SKF96365, $20 \mu \mathrm{m}$; EGTA, 1 mm; U73122, $1 \mu \mathrm{m}$; 2-ApB, $100 \mu \mathrm{m}$; OAG, $50 \mu \mathrm{m}$ ). All the data in these figures are presented as mean \pm SEM of four individual experiments. ${ }^{* * *} p<0.001$ versus control group; ${ }^{\# \# \#} p<0.001$ versus Tat-treated group; ${ }^{+++} p<0.001$ versus both CCL2 and Tat-treated group.

bility. These findings were also confirmed by immunostaining assays using anti-MAP-2 antibody. As shown in Figure 1, $C$ and $D$, loss of neuronal processing and marked loss of MAP-2 immunoreactivity was observed in the Tat-treated group, which was ameliorated by pretreatment of cells with CCL2.

In addition to MAP-2 staining, the neuroprotective effects of CCL2 were further corroborated by morphological analysis of dopaminergic neurons after immunocytochemical staining of the cells with $\mathrm{TH}$, a characteristic marker of these neurons. As shown in Figure 1, E and F, immunocytochemical analysis of untreated rat primary neurons with an anti-TH antibody showed 


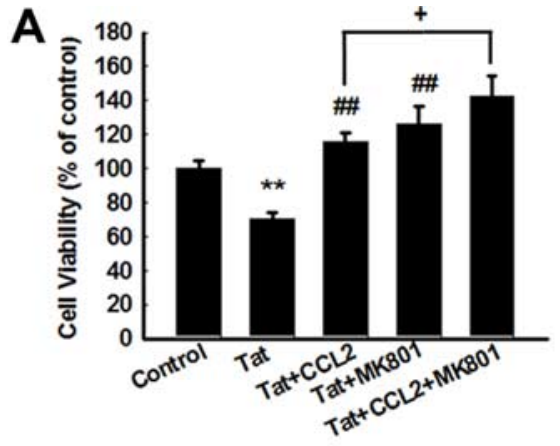

B

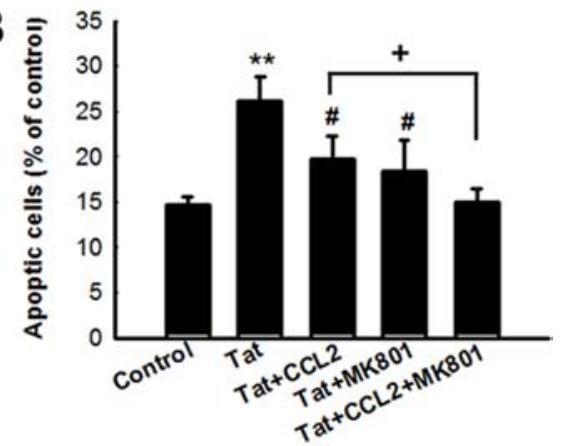

C

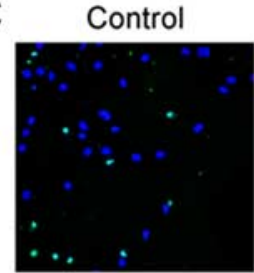

Tat+MK801

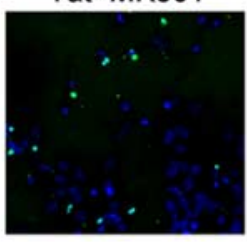

Tat

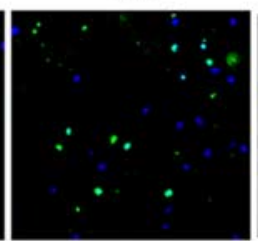

Tat + CCL2

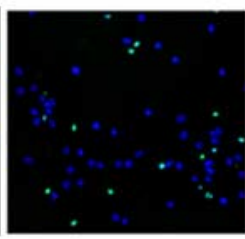

Tat+MK801+CCL2

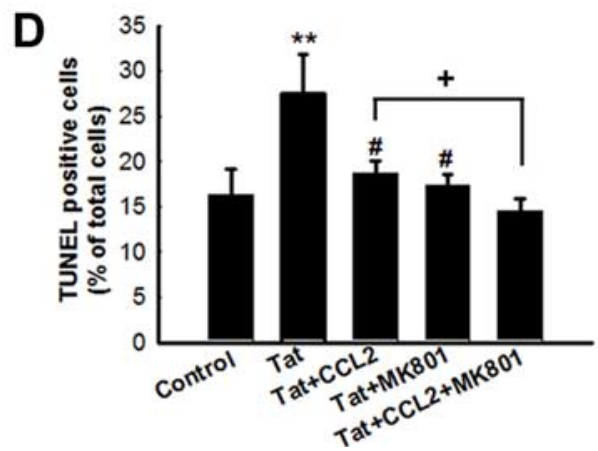

Figure 4. NMDA antagonist MK801 increased CCL2-mediated neuroprotection against Tat. $\boldsymbol{A}$, Pretreatment of neurons with MK801 resulted in increased CCL2-mediated neuroprotection as determined by MTT analysis. Data are presented as mean \pm SEM of four individual experiments. ${ }^{* *} p<0.01$ versus control group; ${ }^{\# \#} p<0.01$ versus Tat-treated group; ${ }^{+} p<0.5$ versus both CCL2 and Tat-treated group. B, Quantification of Hoechst-positive as percentage of total cells. Hoechst-positive cells as percentage of control untreated cells. The data are presented as mean \pm SEM of four individual experiments. All the data in these figures are presented as mean \pm SEM of four individual experiments. ${ }^{* *} p<0.01$ versus control group; ${ }^{*} p<0.05$ versus Tat (200 ng/ml) group; ${ }^{+} p<0.5$ versus both $C \mathrm{CL} 2$ and Tat-treated group. $C$, TUNEL staining of rat primary midbrain neurons treated with Tat and/or CCL2/MK801. D, Quantification of TUNEL-positive cells $(\boldsymbol{C})$ as percentage of total cells. The data are presented as mean \pm SEM of four individual experiments. All the data in these figures are presented as mean \pm SEM of four individual experiments. ${ }^{* *} p<0.01$ versus control group; ${ }^{\#} p<0.05$ versus Tat $(200 \mathrm{ng} / \mathrm{ml})$ group; ${ }^{+} p<0.5$ versus both CCL2 and Tat-treated group.

intense TH-IR neurons (red fluorescence), indicating the presence of elaborate, intact dendrites in the control group. Treatment of neuronal cells with Tat, however, resulted in $\sim 40 \%$ decrease in the total dendrite length per TH-IR neuron. In contrast, pretreatment of neuronal cultures with CCL2 resulted in enhanced TH-IR, indicating rescue of the dendrite length.

Because Tat mediates neurotoxicity by the programmed cell death pathway, we next wanted to examine whether CCL2mediated protection of neurons involved inhibition of Tatinduced neuronal apoptosis. Briefly, primary cultured neurons treated with Tat and/or CCL2 were assessed for apoptosis using Annexin-V binding and propidium iodide uptake. As shown in
Figure $1 G$, there was an increase in Annexin-V-positive cells (22.8 vs $16.7 \%$ ) in Tat-treated cultures compared with control untreated cells as evidenced by flow cytometry. In contrast, CCL2 pretreatment before Tat exposure resulted in decreased numbers of apoptotic cells (22.8 vs $18.7 \%$, Tat treated vs CCL2/Tat)

The above findings were further confirmed by staining cells with Hoechst 33342 , a nuclear dye that specifically stains fragmented nuclei. Untreated neurons exhibited regular and roundshaped nuclei. In contrast, the condensation and fragmentation of nuclei, characteristic of apoptotic cells, was evident in neurons treated with Tat for $24 \mathrm{~h}$ (data not shown). Pretreatment with CCL2 $(100 \mathrm{ng} / \mathrm{ml})$, conversely, reduced Tat-induced neuronal apoptosis, as quantified in Figure $1 \mathrm{H}$.

To corroborate the above findings, TUNEL staining was performed that demonstrated that $25.8 \%$ of cells treated with Tat were TUNEL positive at $24 \mathrm{~h}$, and this increase in cell death was significantly reduced in the presence of CCL2 (Fig. 1I).

\section{Engagement of CCR2 is critical for CCL2-mediated neuroprotection} Because CCL2 mediates signaling via binding to its cognate receptor CCR2, we next examined the expression pattern of CCR2 in rat primary neurons. As shown in Figure $2 A$, rat primary midbrain neurons expressed CCR2 mRNA as demonstrated by reverse transcription (RT)-PCR analysis. We next assessed whether the protective effect of CCL2 was mediated via its binding to its cognate receptor CCR2. As shown in Figure 2B, CCL2 failed to exert neuroprotection in rat primary neurons pretreated with CCR2-selective antagonist RS102895, indicating that the neuroprotection of CCL2 is mediated through CCR2.

To study the relevance of our cell culture findings, we investigated the effects of CCL2 ex vivo using primary neurons isolated from either WT or CCR2 KO mice. As expected, CCL2 protected neurons isolated from WT mice. Conversely, in neurons isolated from CCR2 KO mice, CCL2 failed to exert neuroprotection (Fig. 2C).

\section{TRPC channels are essential for}

\section{CCL2-mediated neuroprotection}

Because $\mathrm{Ca}^{2+}$ is known to mediate cell survival and TRPC is a member of the TRP superfamily that functions as $\mathrm{Ca}^{2+}$ influx channels, it is possible that $\mathrm{Ca}^{2+}$ influx through TRPC channels is required for the neuroprotection mediated by CCL2 2 in primary midbrain neurons. To test this hypothesis, cells were pretreated with the TRPC blocker SKF96365 and assessed for CCL2- 

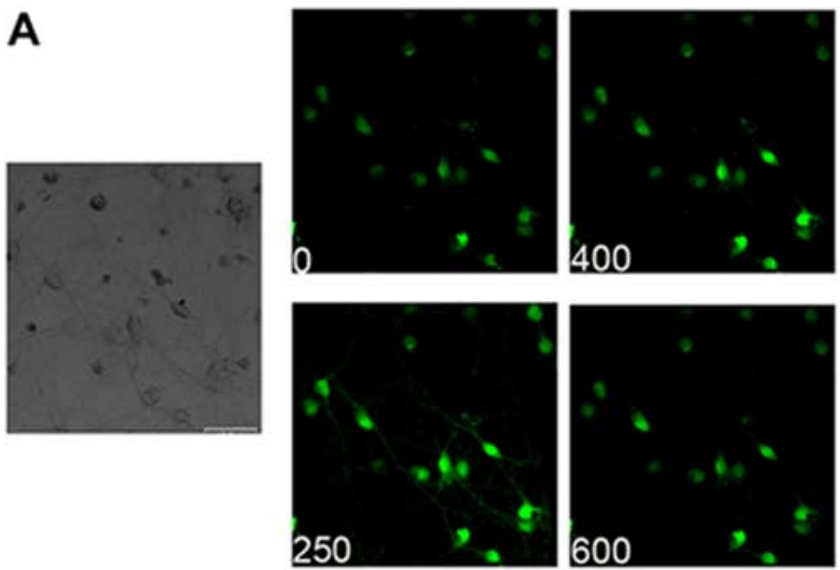

C

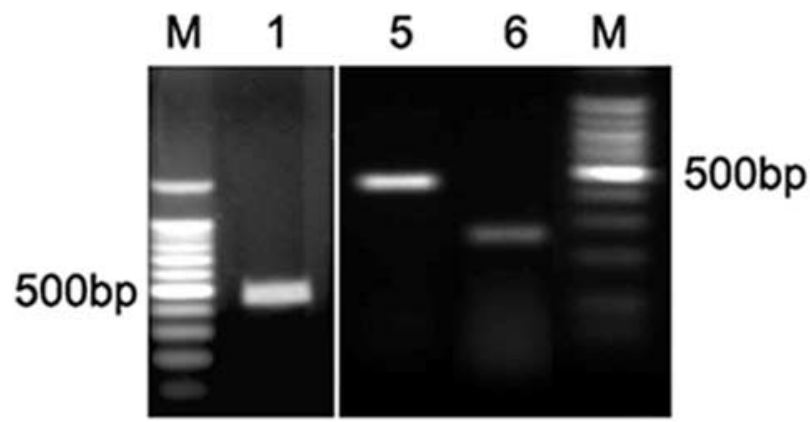

B
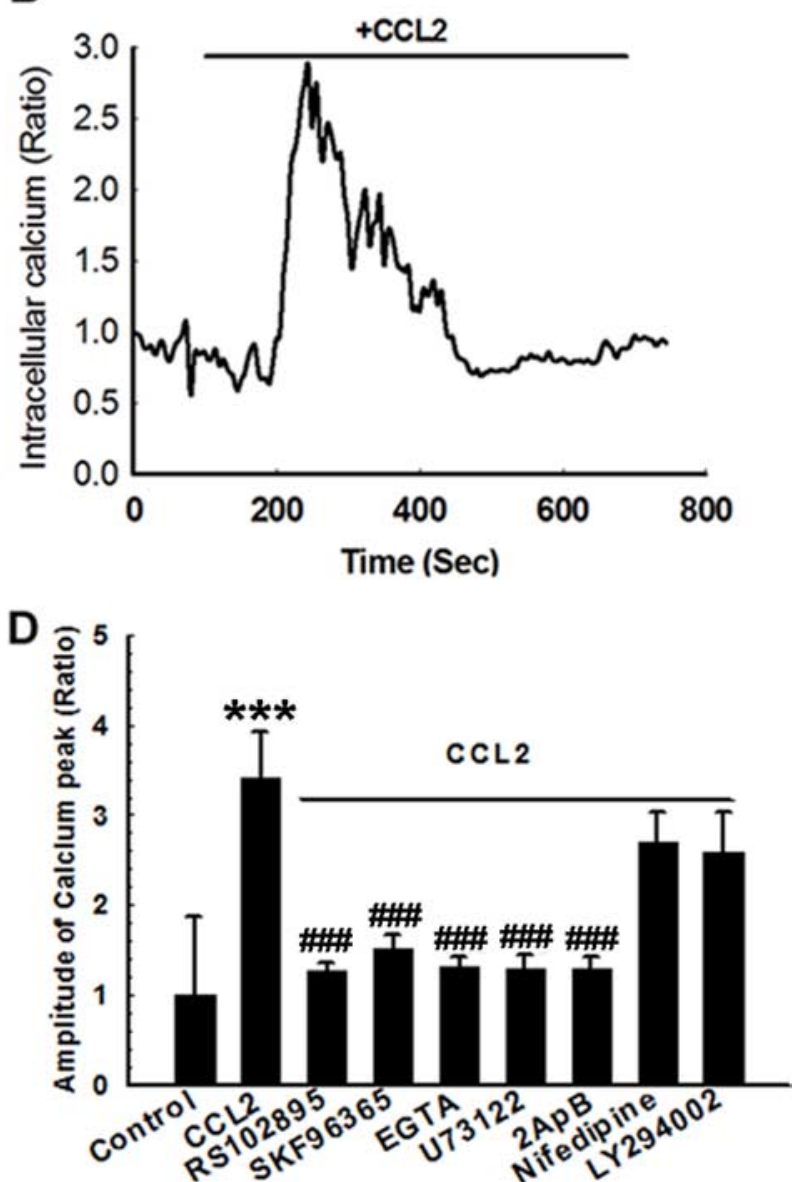

Figure 5. TRPC channels contribute to CCL2-induced intracellular $\mathrm{Ca}^{2+}$ elevations. $\boldsymbol{A}$, Rat primary neurons loaded with Fluo-4 [Ca $\left.{ }^{2+}\right]_{i}$-sensitive fluorophores before and after $C \mathrm{CL} 2$ treatment were recorded within a single field using a Fluoview 300 confocal microscope. Numbers in the panels indicate time in seconds. Scale bar, $10 \mu \mathrm{m} . \boldsymbol{B}$, Changes in intracellular [Ca $\left.{ }^{2+}\right]_{i}$ levels in neurons after CCL2 treatment were measured using the Fluo-4/Fura Red ratio, and the change in ratio is illustrated from a typical neuron. $C$, RT-PCR analysis of TRPC $m$ RNA (1, 5 , and 6) in rat primary neurons. M indicates DNA marker. D, Changes in fluorescence amplitude (Fluo-4/Fura Red) in rat primary neurons exposed to CCL2 in the absence or presence of indicated drugs (RS102895, $20 \mu \mathrm{m}$; SKF96365, $20 \mu \mathrm{m}$; EGTA, 1 mm; U73122, $1 \mu \mathrm{m} ; 2-A p B, 100 \mu \mathrm{m}$; nifedipine, $10 \mu \mathrm{m}$; LY294002, $10 \mu \mathrm{M})$. All the data in these figures are presented as mean \pm SEM of four individual experiments. *** $p<0.001$ versus control group; ${ }^{\# \#} p<0.001$ versus Tat-treated group; ${ }^{+++} p<0.001$ versus both CCL2 and Tat-treated group.

mediated protection against Tat toxicity. As shown in Figure 3, there was inhibition of CCL2-mediated neuroprotection against Tat toxicity in neurons pretreated with SKF96365. These findings underpinned the role of TRPC channels in neuroprotection mediated by CCL2.

Activation of CCR2 by its ligand CCL2 is known to activate PLC, resulting in inonsitol triphophate $\left(\mathrm{IP}_{3}\right)$-dependent release of $\mathrm{Ca}^{2+}$ from intracellular stores and $\mathrm{Ca}^{2+}$ influx from extracellular sources (White et al., 2005). By inference, perturbation of the $\mathrm{PLC} / \mathrm{IP}_{3}$ pathway ought to then suppress the protection exerted by CCL2. As expected, pretreatment of neurons with the PLC inhibitor U73122 did indeed abolish the neuroprotective action of CCL2 against Tat toxicity. The neuroprotective effect of CCL2 also depended on $\mathrm{IP}_{3}$ receptor $\left(\mathrm{IP}_{3} \mathrm{R}\right)$ activation, because exposure of neurons to $\mathrm{IP}_{3} \mathrm{R}$ antagonist, 2-ApB suppressed CCL2-mediated neuroprotection. However, application of OAG, a membrane-permeable analog of DAG, did not protect rat primary midbrain neurons against Tat toxicity, suggesting that the PLC/DAG pathway was not involved in CCL2-mediated neuroprotection. Together, these findings suggested that the $\mathrm{PLC} / \mathrm{IP}_{3} \mathrm{R}$ pathway is required for CCL2-mediated neuronal protection against Tat toxicity.

\section{NMDA antagonist MK801 increased CCL2-mediated} neuroprotection against Tat

Because CCL2 acting via the TRPC channels induces $\mathrm{Ca}^{2+}$ transients, which in turn, lead to neuroprotection, we next wanted to explore whether this mechanism was also critical for protection of neurons against glutaminergic excitoxicity. To validate the role of NMDA receptors in this process, neurons were pretreated with the NMDA receptor antagonist MK801 (+)-5-methyl-10,11dihydro-5H-dibenzo [a,d] cyclohepten-5,10-imine maleate] (10 $\mu \mathrm{M})$ before exposure with CCL2 and/or Tat. As shown in Figure $4 A$, pretreatment of neurons with the NMDA receptor antagonist MK801 for $60 \mathrm{~min}$ increased cell viability by $56 \%$ compared with the Tat-treated group, thus suggesting that glutamatemediated excitotoxicity may be involved in the toxic effects of Tat in primary midbrain neurons. Furthermore, pretreatment of neurons with the NMDA receptor antagonist also resulted in increased neuroprotection mediated by CCL2 against Tat toxicity when compared with neurons not exposed to the antagonist (Fig. 4A). Additional validation of increased neuroprotection in the presence of NMDA receptor antagonist was also performed using Hoechst 33342 and TUNEL staining assays. As shown in Figure $4 B-D$, NMDA receptor antagonist 


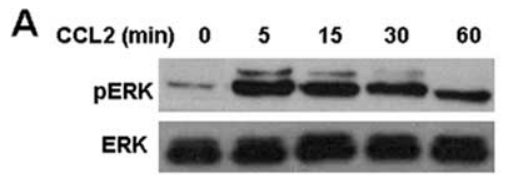

B
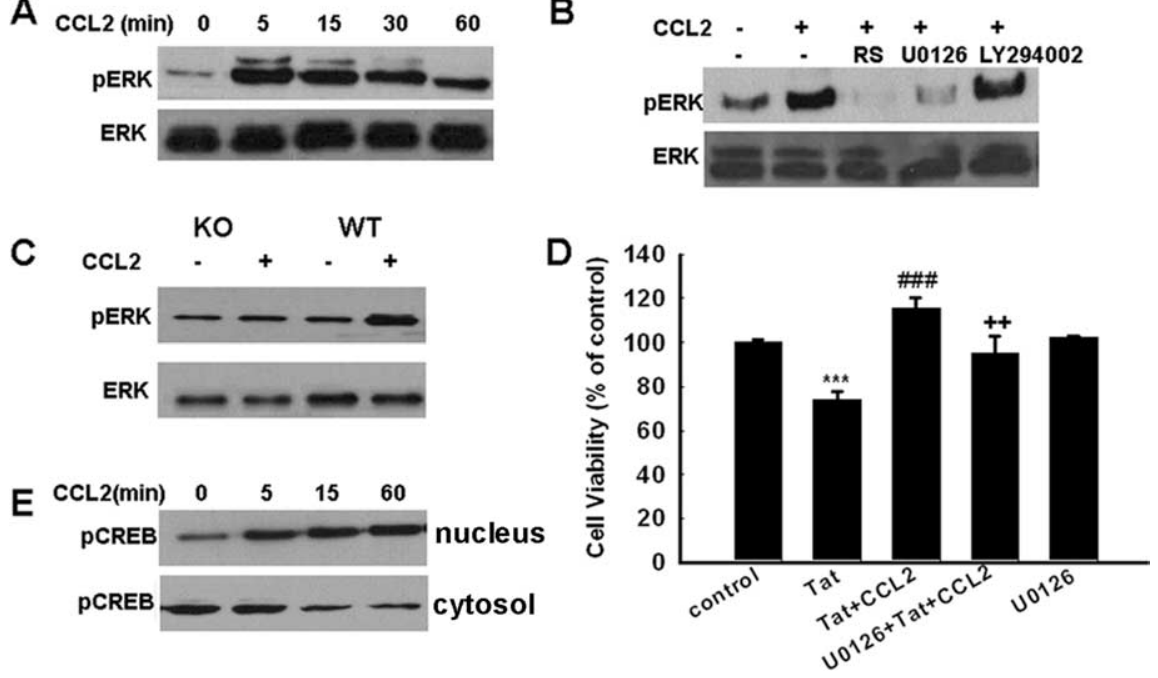

Figure 6. CCL2-induced neuroprotection involves activation of ERK. $A, C C L 2$ induced time-dependent and sustained phosphorylation of ERK. B, CCL2 maintained increased phosphorylation of ERK for at least $1 \mathrm{~h}$, and this effect was abolished by the CCR2 antagonist RS102895, the ERK inhibitor U0126, but not by the PI3K inhibitor LY294002; RS, RS 102895. C, Activation of ERK in rat primary neurons isolated from CCR2 K0 and WT mice treated with CCL2. D, Pretreatment of neurons with U0126 (10 $\mu \mathrm{M})$ for 30 min significantly attenuated the protective effect of $C C L 2$. Data are presented as mean \pm SEM of four individual experiments. ${ }^{* * *} p<0.001$ versus control group; ${ }^{\# \#} p<0.001$ versus Tat-treated group; ${ }^{++} p<0.01$ versus both $\mathrm{CCL} 2$ and Tat-treated group. $E, C C L 2$ exposure resulted in increased time-dependent phosphorylation of CREB in the nuclear fraction with a concomitant decrease in the cytosolic fraction in rat primary neurons.

increased the neuroprotection-mediated by CCL 2 as evidenced by the decreased numbers of apoptotic cells (19 vs 15\%-CCL2/ Tat treated vs MK801/CCL2/Tat in Hoechst staining; 18 vs 14\%CCL2/Tat treated vs MK801/CCL2/Tat in TUNEL staining, respectively).

\section{TRPC channels contribute to CCL2-induced intracellular $\mathrm{Ca}^{2+}$ elevations}

To study the effects of CCL2 on the $\left[\mathrm{Ca}^{2+}\right]_{\mathrm{i}}$ in rat primary midbrain neurons, we first measured CCL2-induced intracellular $\left[\mathrm{Ca}^{2+}\right]_{\mathrm{i}}$ release using Fluo-4 AM imaging. As shown in Figure 5, $A$ and $B$, exposure of neurons to CCL2 triggered a rapid and substantial $\left[\mathrm{Ca}^{2+}\right]_{\mathrm{i}}$ increase in primary midbrain neurons. This response was inhibited by the CCR2 antagonist RS102895 (Fig. $5 D)$. Furthermore, this elevation in $\left[\mathrm{Ca}^{2+}\right]_{\mathrm{i}}$ induced by CCL2 was suppressed by culturing cells in $\mathrm{Ca}^{2+}$-free medium in the presence of EGTA, suggesting thereby that CCL2-induced $\left[\mathrm{Ca}^{2+}\right]_{\mathrm{i}}$ increase depended on the extracellular $\mathrm{Ca}^{2+}$ influx (Fig. $5 D)$.

Because TRPC channels are $\mathrm{Ca}^{2+}$-permeable cation channels, we wanted to assess whether CCL2-induced $\left[\mathrm{Ca}^{2+}\right]_{\mathrm{i}}$ elevations in primary midbrain neurons were mediated by TRPC. It was, however, essential to first investigate the expression pattern of TRPC in rat primary neurons. As demonstrated by RT-PCR analysis and as shown in Figure 5C, rat primary midbrain neurons expressed TRPC1, TRPC5, and TRPC6 but not TRPC2, TRPC3, TRPC4, and TRPC7 (data not shown). To confirm the role of TRPC in CCL2-induced $\left[\mathrm{Ca}^{2+}\right]_{\mathrm{i}}$ elevations, rat midbrain neurons were pretreated with the TRPC inhibitor SKF96365 before treatment with CCL2. As shown in Figure 5D, cells pretreated with the inhibitor demonstrated marked reduction of CCL2induced elevation of $\left[\mathrm{Ca}^{2+}\right]_{i}$, thus underscoring the role of TPRC in this process. In contrast, the L-type voltage-gated calcium channel blocker nifedipine did not affect CCL2-induced $\left[\mathrm{Ca}^{2+}\right]_{\mathrm{i}}$ elevation.
To further dissect the role of PLC activation in CCL2-induced $\left[\mathrm{Ca}^{2+}\right]_{i}$ elevation, pharmacological approach involving the PLC inhibitor U73122 was performed. Similar to findings with the TRPC blocker, neurons cultured in the presence of U73122 failed to demonstrate CCL2induced $\left[\mathrm{Ca}^{2+}\right]_{\mathrm{i}}$ elevation (Fig. 5D). Likewise, treatment of neurons with the $\mathrm{IP}_{3} \mathrm{R}$ antagonist 2-ApB also resulted in inhibition of CCL2-induced $\left[\mathrm{Ca}^{2+}\right]_{\mathrm{i}}$ elevation. As an extension of these studies, we also examined the effect of perturbing PI3K signaling, which was reported to control $\mathrm{Ca}^{2+}$ influx induced by another chemokine, fractalkine. As shown in Figure $5 D$, unlike TRPC and PLC inhibitors, the PI3K inhibitor LY294002 did not affect CCL2induced $\left[\mathrm{Ca}^{2+}\right]_{\mathrm{i}}$ elevation. In summary, CCL2-induced $\left[\mathrm{Ca}^{2+}\right]_{i}$ elevation depended on activation of the $\mathrm{PLC} / \mathrm{IP}_{3} \mathrm{R}$ pathway.

\section{TRPC channels are critical for CCL2- induced ERK/CREB activation}

ERK/mitogen-activated protein kinase pathway has been demonstrated to play a crucial role in antiapoptotic mechanisms. It was therefore of interest to examine the effect of CCL2 on ERK regulation in rat neurons. As shown in Figure 6A, exposure of neurons to CCL2 resulted in a sustained and time-dependent activation of ERK. This effect was blocked by pretreating cells with the CCR2 antagonist RS102895, which significantly attenuated CCL2-mediated sustained activation of ERK (Fig. $6 \mathrm{~B}$ ). The role of CCR2 in activation of ERK was further confirmed in CCR2 $\mathrm{KO}$ neurons wherein CCL2 failed to induce ERK activation, unlike the effect observed in neurons from WT mice (Fig. 6C). Because MEK1/2 lies upstream of ERK, pretreatment of cells with the MAP kinase kinase (MEK) inhibitor U0126 resulted in abrogation of ERK phosphorylation induced by CCL2. The PI3K inhibitor LY294002, conversely, failed to inhibit ERK phosphorylation (Fig. 6B). The functional role of CCL2-induced ERK activation in mediating neuroprotection was also corroborated using cell viability assays, wherein CCL2 failed to exert its protective effect in cells pretreated with U0126, thereby underscoring the role of this pathway in CCL2-mediated neuroprotection (Fig. $6 D$ ). Because CREB is a transcriptional factor that lies downstream of ERK and plays an important role in cell survival, we next examined CREB phosphorylation by Western blot analysis. As shown in Figure 6E, CCL2-mediated increase in nuclear CREB phosphorylation correlated well with a concomitant decrease in the cytosolic compartment.

Having determined the role of TRPC channels in CCL2mediated neuroprotection, we next wanted to dissect the role of these channels in CCL2-mediated ERK activation pathway. As shown in Figure $7 A$, pretreatment of neurons with the TRPC inhibitor SKF96365 markedly attenuated CCL2-induced phosphorylation of both ERK and CREB. Furthermore, blocking of PLC and $\mathrm{IP}_{3} \mathrm{R}$ using the respective inhibitors U73122 and 2-ApB also resulted in reduced phosphorylation of ERK and CREB. Next, using the time-dependent study, it was also demonstrated that CCL2-induced ERK (Fig. 7B) and CREB (Fig. 7C) phosphorylation was sensitive to SKF96365 even after $60 \mathrm{~min}$ of exposure 
A
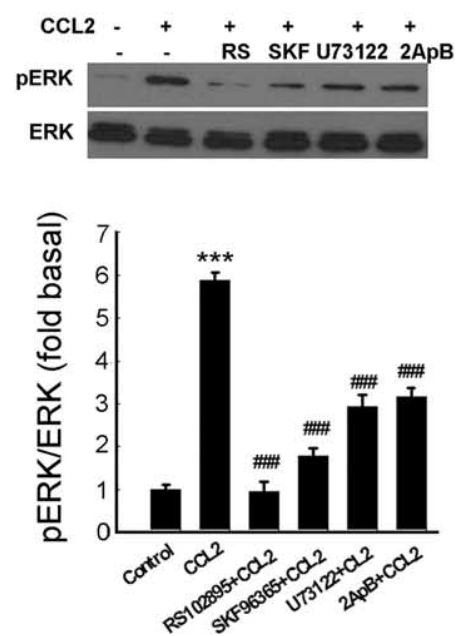

B
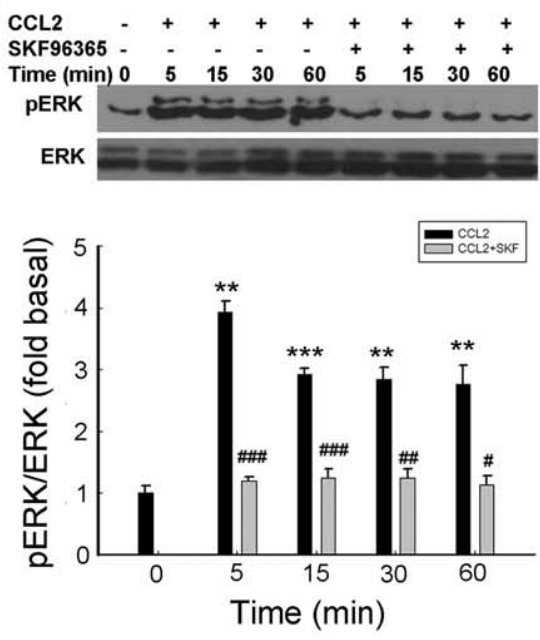
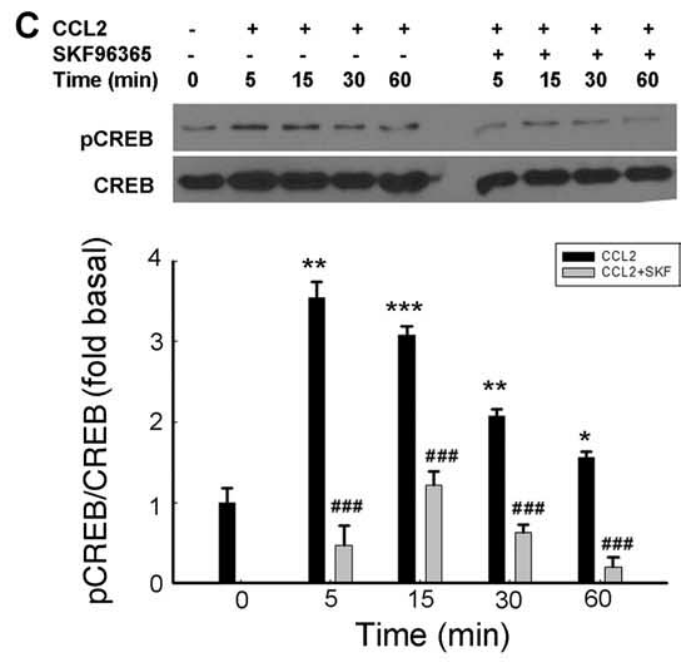

Figure 7. TRPC channels are critical for CCL2-induced ERK/CREB activation. $\boldsymbol{A}$, Rat primary neurons exposed to CCL2 in the presence or absence of indicated agents were monitored for CCL2-mediated ERK activation [RS102895 (RS), $20 \mu \mathrm{m}$;SKF96365 (SKF), $20 \mu \mathrm{m}$;U73122, $10 \mu \mathrm{m} ; 2$-ApB, $100 \mu \mathrm{m}$ ]. Densitometric analysis of pERK/ERK from a representative immunoblot is presented. Data are presented as mean \pm SEM of four individual experiments. ${ }^{* * *} p<0.001$ versus control group; ${ }^{\# \# ~} p<0.001$ versus CCL2 group. $\boldsymbol{B}, \boldsymbol{C}$, Rat primary neurons exposed to CCL2 in the presence or absence of the TRPC blocker SKF96365 (20 $\mu \mathrm{m})$ were monitored for CCL2-mediated ERK $(\boldsymbol{B})$ and CREB $(\boldsymbol{C})$ phosphorylation. Densitometric analyses of pERK/ERK or pCREB/CREB from the representative immunoblots are presented. Data are presented as mean \pm SEM of four individual experiments. ${ }^{*} p<0.05,{ }^{* *} p<0.01,{ }^{* * *} p<0.001$ versus control group; $p<0.05,{ }^{\# \#} p<0.01$, $\# \#$ \#\# 0.001 versus CCL2 group.

to CCL2. In summary, these findings suggested that ERK and CREB phosphorylation triggered by CCL2 depended on the TRPC/IP ${ }_{3}$ R pathway.

To further confirm the role of specific TRPC protein(s) in CCL2-induced ERK phosphorylation, each of the TRPC subtypes $(1,5$, or 6$)$ was individually downregulated using specific siRNA, followed by assessment of CCL2-induced ERK and CREB phosphorylation. For each TRPC protein, at least three siRNA sequences were designed and the one with the highest efficiency and specificity was selected. It was found that siRNA for TRPC1 caused a marked reduction in the expression of TRPC 1 protein, without influencing the expression of TRPC5 and TRPC6. Similarly, siRNAs against TRPC5 or TRPC6 decreased the expression of TRPC5 or TRPC6, respectively (Fig. 8A). Interestingly, siRNA for both TRPC1 and TRPC5 decreased CCL2-mediated ERK (Fig. $8 B, C$ ) and CREB (Fig. 8D,E) phosphorylation, whereas TRPC6 siRNA exerted no such effect. These findings thus underpinned the roles of TRPC 1 and TRPC5 in CCL2-mediated ERK and CREB phosphorylation.

\section{TRPC channels are not essential for} CCL2-induced Akt/NF- $\kappa$ B activation In addition to the MEK/ERK pathway, the PI3K/Akt pathway plays a critical role in cell survival. To dissect the role of this pathway in CCL2-mediated neuroprotection, cell lysates were examined for phosphorylation of Akt. As shown in Figure 9A, after exposure of neurons to CCL2, there was an enhanced and

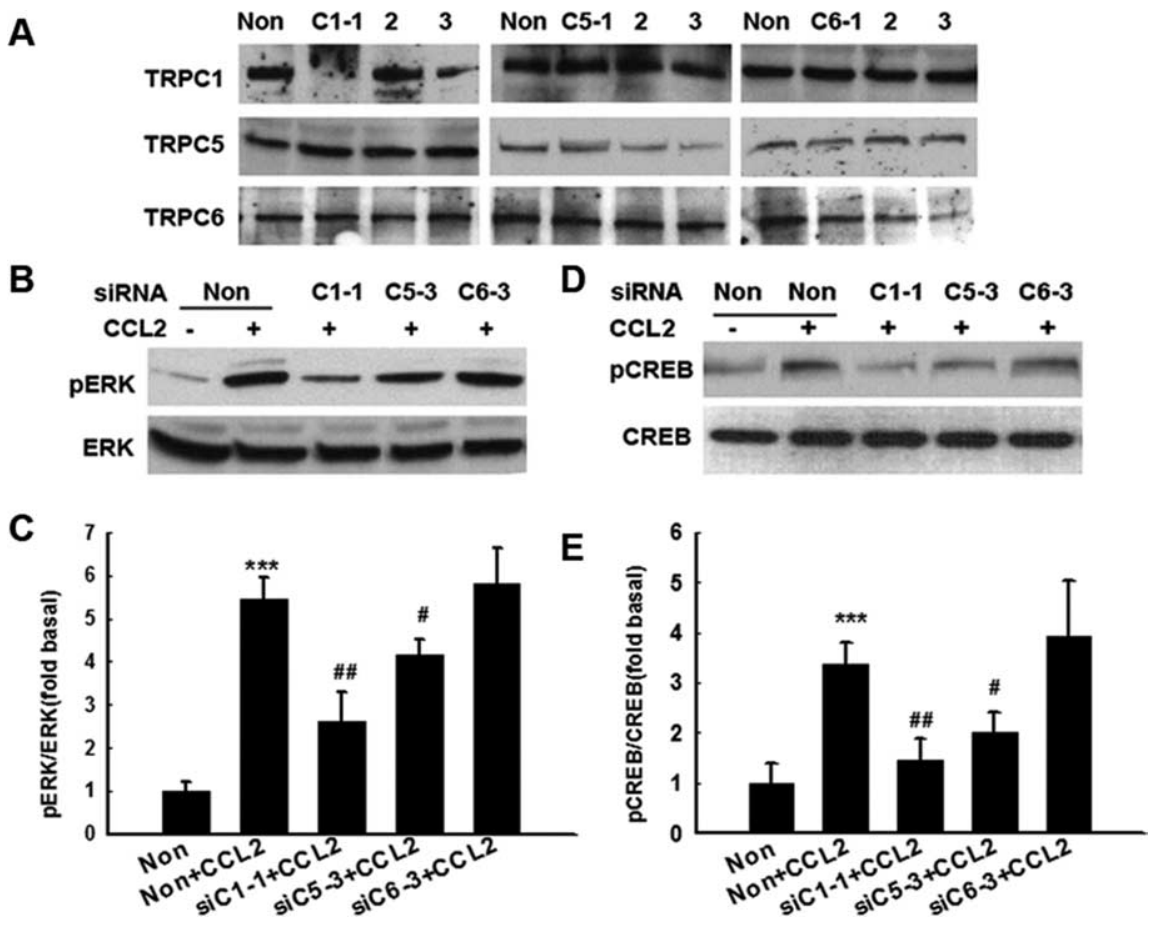

Figure 8. TRPC1 and TRPC5 are essential for CCL2-mediated phosphorylation of ERK/CREB. $A$, Western blot analysis of wholecell lysates from rat primary midbrain neurons transfected with siRNAs against TRPC1 (C1-1 to 3), TRPC5 (C5-1 to 3), TRPC6 (C6-1 to 3), or nonsense (Non) siRNA using antibodies specific for TRPC1, TRPC5, or TRPC6. Data are representative of three independent experiments. $\boldsymbol{B}, \boldsymbol{D}$, Effects of TRPC1, TRPC5, and TRPC6 suppression by siRNAs on CCL2-mediated ERK and CREB phosphorylation. $C, E$, Densitometric analyses of CCL2-mediated phosphorylation of ERK or CREB in the presence or absence of siRNAs. Data are presented as mean \pm SEM of four individual experiments. ${ }^{* * *} p<0.001$ versus control group; ${ }^{\#} p<0.05,{ }^{\# \#} p<$ 0.01 versus CCL2 group. Data are representative of three independent experiments.

sustained activation of Akt, an effect that was abrogated by the CCR2 antagonist RS102895 (Fig. 9B). The role of CCR2 in CCL2mediated activation of Akt was further confirmed by failure of the chemokine to activate Akt in CCR2 KO neurons compared with the WT cells (Fig. 9C). Because PI3K lies upstream of Akt, pre- 
A
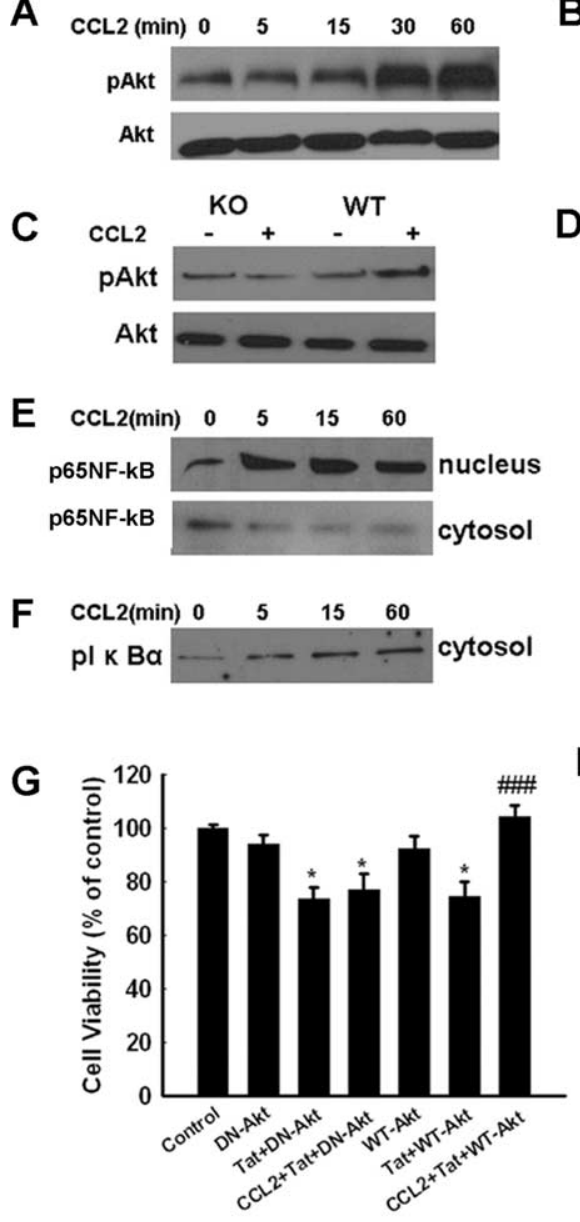

B

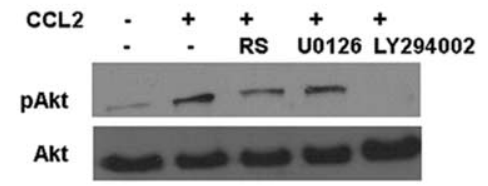

D
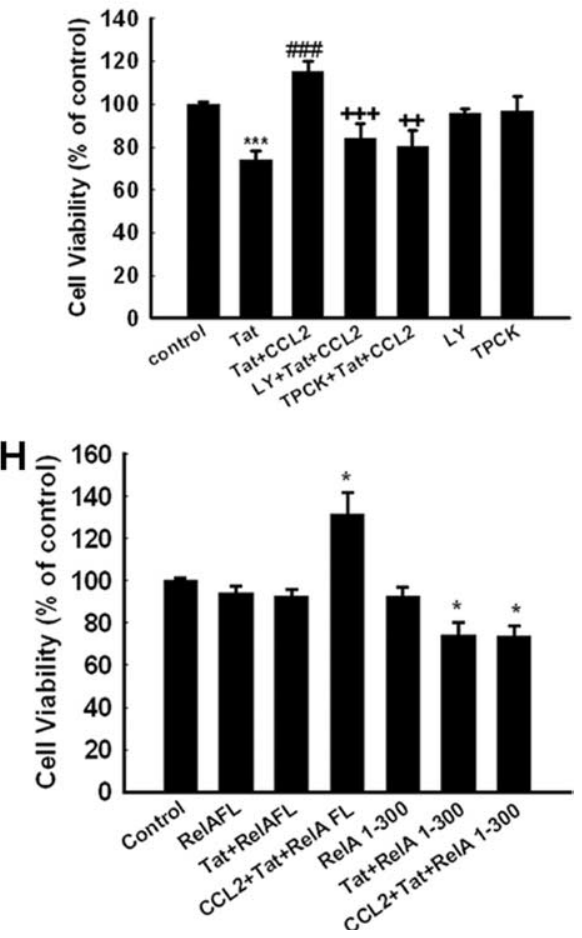

Figure 9. Role of $\mathrm{PI3K} / \mathrm{Akt}$ pathway in $\mathrm{CCL} 2$-mediated neuroprotection in rat primary midbrain neurons. $\boldsymbol{A}, \mathrm{CCL} 2$ induced time-dependent activation of Akt. $\boldsymbol{B}, \mathrm{CCL} 2$ maintained sustained phosphorylation of Akt for at least $1 \mathrm{~h}$, and this effect was attenuated by both the CCR2 antagonist RS102895 and the PI3K inhibitor LY294002 but not by the MEK inhibitor U0126. C, Activation of Akt in rat primary neurons isolated from CCR2 K0 and WT mice treated with CCL2. D, Pretreatment of neurons with LY294002 and TPCK resulted in inhibition of CCL2-mediated protection using MTT analysis. Data are presented as mean \pm SEM of four individual experiments. ${ }^{* *} p<0.001$ versus control group; ${ }^{\# \# \#} p<0.001$ versus Tat-treated group; ${ }^{++} p<0.01$, $+++p<0.001$ versus both CCL2 and Tat-treated group. $\boldsymbol{E}$, Exposure of rat neurons to CCL2 resulted in increased phosphorylation of the p65 subunit of NF- $\kappa$ B level in the nuclear fraction with a concomitant decrease in the cytosolic fraction. $F$, CCL2 induced increased phosphorylation of $\mathrm{I}_{\kappa} \mathrm{B} \alpha$ in the cytosolic fraction of neurons exposed to the chemokine. $\mathbf{G}$, Infection of neurons with the dominant-interfering Akt (DN-Akt) resulted in abrogation of CCL2-mediated neuroprotection. Infection with WT Akt (WT-Akt) construct had no effect. Data are presented as mean \pm SEM of four individual experiments. ${ }^{*} p<0.05$ versus adenovirus alone group; ${ }^{\# \# \# ~}<0.001$ versus Tat-treated group. $\boldsymbol{H}, 0$ verexpression of full-length $p 65 /$ RelA NF- $\kappa$ B construct in rat neurons resulted in CCL2-mediated neuroprotection. RelA mutant constructs abrogated CCL2-mediated protection against Tat toxicity. The data are presented as mean \pm SEM of four individual experiments. ${ }^{*} p<0.05$ versus adenovirus alone group.

treating cells with the PI3K inhibitor LY294002 resulted in the inhibition of CCL2-mediated activation of Akt. In contrast, the MEK inhibitor U0126 failed to inhibit CCL2-mediated Akt activation (Fig. 9B). We next wanted to address the functional role of $\mathrm{PI} 3 \mathrm{~K} / \mathrm{Akt}$ in the neuroprotection of CCL2 using a pharmacological approach. As shown in Figure 9D, Tat neurotoxicity was completely inhibited by pretreatment of cells with CCL2 in the absence of the PI3K inhibitor LY294002. In contrast, in neurons cultured in the presence of LY294002, CCL2 was not able to rescue the cells from Tat-mediated toxicity. These results suggested that the PI3K/Akt pathway is involved in the antiapoptotic effects of CCL2 in primary neurons. Additional confirmation of the involvement of the Akt pathway in CCL2-mediated neuroprotection was conducted using an adenovirus vector encoding a dominant-interfering form of Akt. As shown in
Figure $9 G$, in neurons infected with the dominant-interfering form of Akt, CCL2 failed to protect against Tat toxicity. Infection of neurons with wild-type Akt, conversely, resulted in CCL2mediated neuroprotection.

Akt can exert its antiapoptotic action by regulating the activity of several downstream effectors, such as the transcription factor NF- $\kappa \mathrm{B}$. In the cell, NF- $\kappa \mathrm{B}$ activity and nuclear translocation are prevented by inhibitory nuclear factor $\kappa \mathrm{B}$ inhibitor $(\mathrm{I} \kappa \mathrm{B})$ proteins, whose inactivation/degradation are regulated by their phosphorylation. Thus, a reduction in the cytosolic level of $\mathrm{I} \kappa \mathrm{B}$ and an increase in its phosphorylated form are generally induced by stimuli that cause NF- $\kappa \mathrm{B}$ activation. Interestingly, as shown in Figure 9E, treatment of neurons with CCL2 resulted in increased nuclear translocation of the p65/ RelA subunit of NF- $\kappa \mathrm{B}$ with a concomitant reduction in the cytoplasmic level of its inhibitory protein $\mathrm{I} \kappa \mathrm{B}$ (Fig. $9 F$ ). Nuclear translocation of NF- $\kappa$ B p65/RelA was also demonstrated by immunocytochemistry in CCL2-treated neurons (data not shown).

Confirmation of the role of NF- $\kappa \mathrm{B}$ in CCL2-mediated neuroprotection was performed using Adeno-RelA-GFP constructs. Almost $40 \%$ of primary neurons were infected with RelA (data not shown). In neurons infected with full-length p65/ RelA construct, Tat treatment failed to exert demonstrable cell toxicity, and CCL2 exposure resulted in enhanced cell survival, as expected, compared with the control group. In contrast, in neurons infected with the 1-300 mutant p65/RelA construct, Tat was neurotoxic, and CCL2 treatment failed to rescue cells from Tat toxicity (Fig. 9H).

Having determined that TRPC channels were involved in regulation of ERK/ CREB activation, we next wanted to dissect the role of these channels in the CCL2mediated Akt activation pathway. As shown in Figure $10 \mathrm{~A}$, pretreatment of neurons with the TRPC channel blocker SKF96365 did not attenuate CCL2-induced Akt activation. Furthermore, a timedependent study demonstrated that both CCL2-induced phosphorylation of Akt (Fig. $10 \mathrm{~B}$ ) and NF- $\kappa$ B translocation (Fig. 10C) were insensitive to TRPC blocker even after $60 \mathrm{~min}$ of exposure to CCL2. Interestingly, unlike the ERK/CREB phosphorylation, TRPC siRNAs failed to abrogate Akt phosphorylation and NF- $\kappa \mathrm{B}$ translocation in the presence of CCL2 as shown in Figure $10 \mathrm{D}$. These findings suggest that CCL2-mediated Akt phosphorylation and NF- $\kappa \mathrm{B}$ translocation are independent of TRPC.

Protective role of CCL2/CCR2 axis against Tat-induced dopaminergic neuronal loss in the substantia nigra One of the hallmark neuropathological features of HAD is the loss of nigrostriatal neurons (Itoh et al., 2000). To determine the 
protective effect of CCL2 on dopaminergic neurons, WT and CCR2 KO mice were injected with either saline or CCL2 in the striatum followed by HIV-1 Tat microinjection and was examined $7 \mathrm{~d}$ later for $\mathrm{TH}$ positive neurons in the susbtantia nigra region of the brain. As shown in Figure $11 \mathrm{~A}$, as expected, there was increased neuronal loss in the Tat-injected WT mice, as evidenced by decreased $\mathrm{TH}$ staining. Interestingly, there was a greater reduction of $\mathrm{TH}$ positive neurons in the substantia nigra of Tat-injected CCR2 KO mice. Although CCL2-mediated protection in the WT mice (as evidenced by increased $\mathrm{TH}$ positive neurons), there was no protection against Tat in CCR2 KO mice as quantified in Figure $11 B$.

\section{Discussion}

CCL2 is a constitutively expressed chemokine in the normal brain that is produced by a variety of CNS cells. It has been demonstrated that CCL2 can function not only to recruit monocytes into the CNS of HIV-1 patients but can also play a critical role in protection of fetal neurons against HIV-1 Tat toxicity (Eugenin et al., 2003). Although CCL2 has primarily been linked to the deleterious effects associated with the pathology of HAD (Eugenin et al., 2006), many other beneficial roles of this chemokines are being increasingly documented. These include its neurotrophic (Bolin et al., 1998), neuromodulatory (Gosselin et al., 2005), and neurohormonal actions. Similarly, newer pleiotrophic roles of the chemokine fractalkine are also becoming increasingly appreciated in the literature. Specifically, although fractalkine is a known chemoattractant, it can also function to regulate neuronal survival via its antiapoptotic effects (Meucci et al., 1998, 2000).

Nigrostriatal neurons are susceptible to HIV-1 proteinmediated toxicity, and HIV-1 Tat is known to inhibit TH gene expression in dopaminergic neuronal cells, contributing to motor abnormalities in HAD patients (Zauli et al., 2000). Consistent with the previous study, data presented here provide strong evidence that CCL2 can act directly on midbrain neurons and activate distinct and related neuronal survival pathways. It has been shown by Banisadr et al. (2005) that CCR2 localized with the dopaminergic neurons in the substantia nigra pars compacta, thereby implicating its role in neuronal communication and possibly in dopaminergic neurotransmission circuitry. In our present studies, we detected the presence of CCR2 mRNA in rat primary cultured midbrain neurons. Additionally, using both pharmacological as well as genetic approaches, we demonstrated that CCL2-mediated neuroprotection involved activation of its cognate receptor CCR2.

A novel finding of this study is the role of TRPC in neuroprotection induced by CCL2, which lends credence to previous reports indicating that TRPC signaling contributes to neuroprotec- tion (Bollimuntha et al., 2005; Jia et al., 2007). The mammalian TRPC channel family consists of seven members, TRPC1TRPC7, that appear to function as receptor-operated channels, analogous to the TRP channels involved in Drosophilia phototransduction (Clapham et al., 2001). With the exception of TRPC2, these channels are widely distributed in the mammalian brain. Consistent with the previous reports demonstrating the colocalization of TRPC $(1,5$, and 6$)$ with the dopaminergic neurons in substantia nigra, our findings also provide evidence for the presence of these three TRPC isoforms in primary midbrain neurons (Bollimuntha et al., 2006; De March et al., 2006; Martorana et al., 2006). The mammalian TRPC channels can be activated by G-protein-coupled receptors and receptor tyrosine kinases (Clapham, 2003; Ramsey et al., 2006; Ambudkar and Ong, 2007). CCR2 is a G-protein-coupled receptor that, during binding to its ligand CCL2, results in activation of PLC, leading to hydrolysis of phosphatidylinositol-4, 5-biphosphate into membrane-bound DAG and soluble $\mathrm{IP}_{3}$. Generation of $\mathrm{IP}_{3}$ results in $\mathrm{IP}_{3}$ receptormediated release of $\mathrm{Ca}^{2+}$ from intracellular stores as well as $\mathrm{Ca}^{2+}$ influx from outside the cell (White et al., 2005). 
A

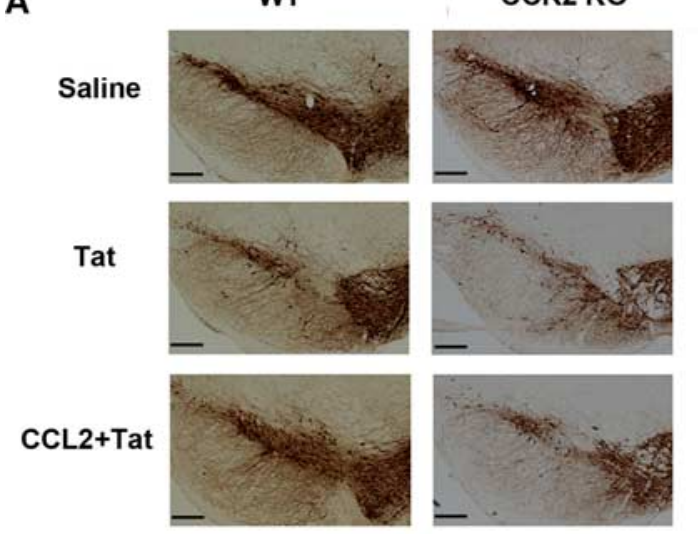

B

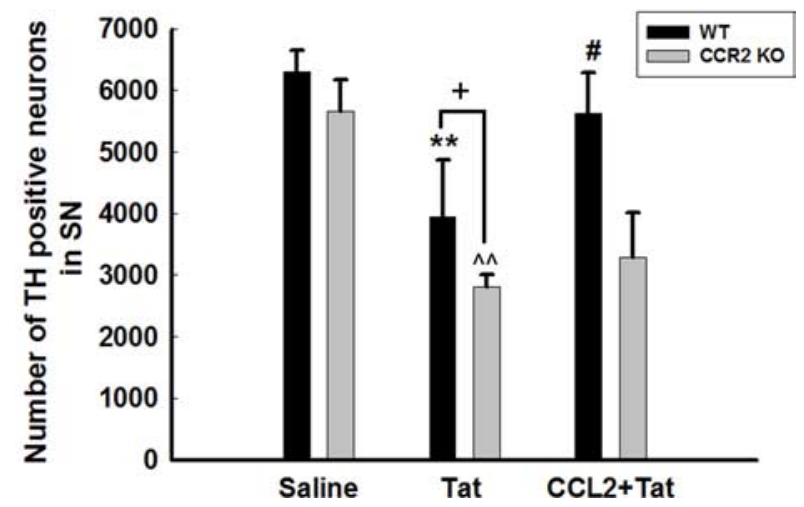

Figure 11. Protective role of CCL2/CCR2 axis against Tat-induced dopaminergic neuronal loss in the substantia nigra. Representative mesencephalic sections from WT and CCR2 KO mice microinjected with either saline or Tat in the presence or absence of $\mathrm{CCL} 2$ were examined $7 \mathrm{~d}$ later for TH-positive neurons. Fewer SNc TH-positive neurons survived in Tat-treated CCR2 KO mice compared with WT littermates. CCL2 exerted neuroprotection in WT but not CCR2 K0 mice. SNc neurons (means \pm SEM from 4 mice per group) were counted by stereology. ${ }^{* *} p<0.01$ versus saline WT mice; $p<0.01$ versus saline $K 0$ mice; ${ }^{*} p<0.05$ versus Tat-injected WT mice; ${ }^{+} p<0.05$ versus Tat-injected WT mice. Scale bars, $300 \mu \mathrm{m}$.

To further explore the robustness of the neuroprotective role of CCL2, we next sought to assess whether CCL2 could also limit glutamatergic excitotoxicity induced by Tat. The NMDA receptor antagonist MK801 not only ameliorated neurotoxicity caused by Tat but also increased the neuroprotection-mediated by CCL2. A possible explanation for this protection could be the possible activation of NMDA receptor by Tat, an effect that has been well documented previously (Haughey et al., 2001; Song et al., 2003). These findings are consistent with the report by Eugenin et al. (2003) demonstrating that Tat-mediated increase of extracellular glutamate levels and NMDA receptor expression could be reversed by pretreatment of neurons with CCL2.

CCL2 triggers $\left[\mathrm{Ca}^{2+}\right]_{\mathrm{i}}$ transients in primary cultures of cortical, hippocampal, hypothalamus, and mesencephalon neurons (Banisadr et al., 2005). Although there is evidence that CCL2 induces an increase of $\left[\mathrm{Ca}^{2+}\right]_{\mathrm{i}}$, the mechanisms underlying this elevation remain unclear. We report herein that CCL2 induces $\left[\mathrm{Ca}^{2+}\right]_{\mathrm{i}}$ elevation through its receptor CCR2, because CCR2 antagonist abolished CCL2-mediated $\left[\mathrm{Ca}^{2+}\right]_{i}$ elevations. Furthermore, CCL2-mediated increase in $\left[\mathrm{Ca}^{2+}\right]_{\mathrm{i}}$ transients was primarily attributable to $\mathrm{Ca}^{2+}$ entry through the $\mathrm{PLC} / \mathrm{IP}_{3}$-induced $\mathrm{Ca}^{2+}$ release and the TRPC channels. These findings were supported by the use of pharmacological inhibitors, including U73122 (PLC inhibitor), 2-ApB ( $\mathrm{IP}_{3} \mathrm{R}$ antagonist), and SKF96365 (TRPC blocker), all of which blocked CCL2-mediated $\left[\mathrm{Ca}^{2+}\right]_{\mathrm{i}}$ eleva-

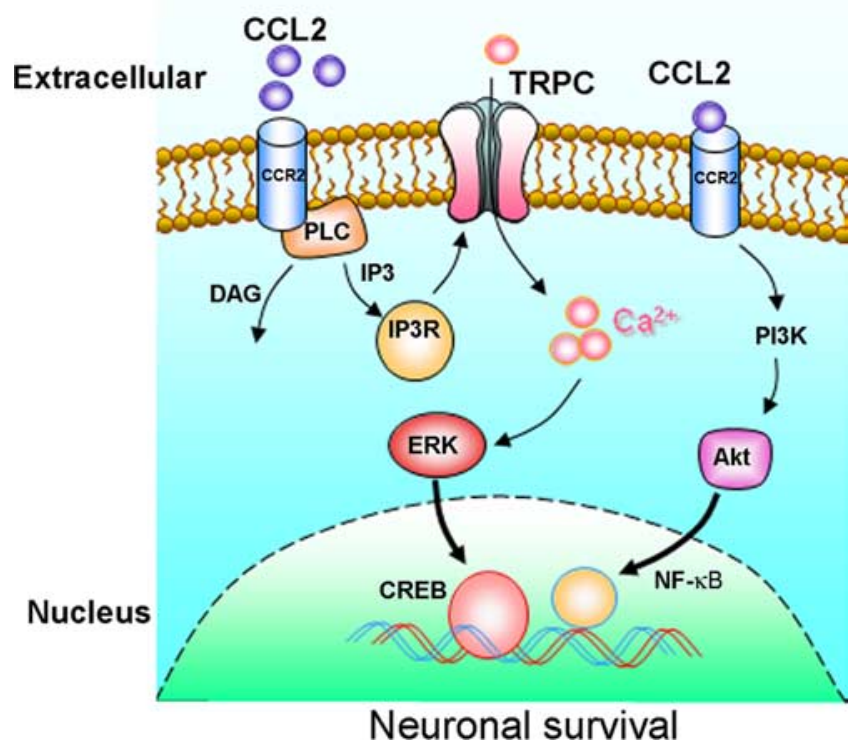

Figure 12. Schematic illustration demonstrating signaling pathways involved in CCL2mediated neuroprotection in rat primary neurons. CCL2 binding to its cognate CCR2 receptor stimulates the $P L C / P_{3} R$ pathway, which in turn activates TRPC channels, resulting in elevation of $\left[\mathrm{Ca}^{2+}\right]_{i}$ transients. $\left[\mathrm{Ca}^{2+}\right]_{i}$ elevation then results in activation of MEK/ERK pathways, leading to CREB activation and consequently neuronal survival. In addition to this, CCL2 binding to CCR2 can also activate another distinct pathway, PI3K/Akt/NF- $\kappa$ B, which can also lead to potentiation of neuronal survival.

tions. Calcium influx from extracellular source was confirmed by EGTA, an extracellular $\mathrm{Ca}^{2+}$ chelator, which also blocked CCL2mediated elevation of $\left[\mathrm{Ca}^{2+}\right]_{i}$. Therefore, CCR2 engagement by CCL2 results in downstream signaling targeting activation of TRPC channels. Interestingly, PI3K inhibitor had no effect on CCL2-induced $\left[\mathrm{Ca}^{2+}\right]_{\mathrm{i}}$ elevations. Our findings are in contrast to the reports by Ré and Przedborski (2006) demonstrating involvement of PI3K-dependent $\mathrm{Ca}^{2+}$ influx after fractalkine binding to its G-protein-coupled receptor (CX3CR1).

The seven TRPC channels are divided into two groups based on their homology and mechanisms of activation. TRPC1, TRPC4, and TRPC5 have been characterized as store-dependent channels, TRPC3, TRPC6, and TRPC7 are the store-independent channels, and TRPC3, TRPC5, and TRPC7 are recognized to function as both receptor- and store-operated channels (Chaudhuri et al., 2008). The present study showed that TRPC1 and TRPC 5 but not TRPC6 are required for CCL2-mediated neuroprotection. This is consistent with the previous findings demonstrating the protective role of TRPC1 in 1-methyl-4-phenyl1,2,3,6-tetrahydropyridine (MPTP)-induced toxicity in SHSY5Y cells (Bollimuntha et al., 2006). The precise mechanisms by which $\mathrm{Ca}^{2+}$ regulates neuronal survival, however, remain poorly understood. Recent studies have shed light on the mechanisms underlying the activation of TRPC channels by the growth factor BDNF. For example, activation of TRPC 3 and TRPC6 by BDNF stimulates two signaling pathways: $\mathrm{Ca}^{2+} / \mathrm{Ras} / \mathrm{MEK} / \mathrm{ERK}$ and $\mathrm{Ca}^{2+} / \mathrm{CaM} /$ calcium/calmodulin-dependent kinase, which converge on CREB activation (Jia et al., 2007). Additional studies are required to examine the downstream signaling pathways of TRPC channels to enhance neuronal survival. In addition to the ERK/CREB pathway, PI3K/Akt activation was also involved in CCL2-mediated neuroprotection; blocking TRPC channels, however, had no effect on Akt activation.

The CCL2/CCR2 axis was linked to the activation of ERK and 
PI3K/Akt pathways, an effect that is consistent with the previous reports demonstrating that CCL2 stimulates ERK and PI3K/Akt pathways in various other cell types (Kim et al., 2002; Sodhi and Biswas, 2002; Jimenez-Sainz et al., 2003). A similar pathway has been reported for another chemokine, fractalkine, which regulates $\left[\mathrm{Ca}^{2+}\right]_{\mathrm{i}}$ levels, synaptic transmission, and activation of ERK/CREB and the PI3K/Akt pathways in hippocampal neurons (Meucci et al., 1998, 2000). The ERK pathway can be activated via PI3K activation or independent of it, depending on the cell type (Heo and Han, 2006). In the present study, CCL2-mediated neuroprotection via ERK involved the PI3K-independent pathway because PI3K inhibitor failed to inhibit CCL2-mediated ERK phosphorylation. The role of the Akt pathway in the neuroprotective effects of CCL2 was further confirmed using a dominantinterfering form of Akt. Activation of NF- $\kappa \mathrm{B}$ plays a key role in enhancing neuronal survival after exposure to a wide array of stimuli (Maggirwar et al., 1998; Glazner et al., 2000; Ramirez et al., 2001). Akt transduces its signal via activation of its downstream transcription factor NF- $\kappa \mathrm{B}$, an effect that has been implicated in fractalkine-mediated neuroprotection (Meucci et al., 2000). Consistent with these findings, we also report the role of p65/RelA nuclear translocation in CCL2-mediated neuronal survival.

To investigate the relevance of CCL2-mediated protection in vivo, a genetic approach using the CCR2 $\mathrm{KO}$ mice was used. Intrastriatal microinjection of Tat has been well documented to cause dopaminergic neuronal loss in the substantia nigra (Zauli et al., 2000). Consistent with this, we also demonstrated that microinjection of HIV-1 Tat into the striatum resulted in increased dopaminergic neuronal loss in the substantia nigra. Interestingly, Tat microinjection into the CCR2 KO mice resulted in exacerbated dopaminergic neuronal loss compared with the WT mice, thus confirming the protective role of CCL2/CCR2 axis against HIV-1 Tat toxicity in vivo. These findings are in agreement with the neuroprotective role of CCL 2 in the MPTP toxicity model (Kalkonde et al., 2007). The protective role of CCR2 has also been shown in the Alzheimer's-like disease model, wherein CCR2 deficiency has been shown to impair early protective phase of microglial accumulation and thus accelerate progression of the disease (El Khoury et al., 2007). Interestingly, CCL2-mediated neuroprotection against Tat in the WT mice but not the CCR2 $\mathrm{KO}$ mice further corroborated the role of the CCL2/CCR2 axis in maintenance of the dopaminergic neuronal integrity. Whether such a neuroprotective effect is limited to specific anatomical regions of the brains remains to be explored.

In summary, activation of CCR2 by CCL2 results in stimulation of $\mathrm{PLC} / \mathrm{IP}_{3} \mathrm{R}$ pathway, leading to activation of TRPC channels, which in turn, can cause elevation of $\left[\mathrm{Ca}^{2+}\right]_{\mathrm{i}}$ transients and subsequent activation of ERK/CREB pathway but not the Akt/ NF- $\kappa$ B pathway (as summarized in Fig. 12). Overall, these studies determine the distinct roles of TRPC channels in CCL2-mediated neuroprotection against Tat toxicity and create a paradigm shift in our thinking of how chemokines can exert paradoxical roles within the CNS.

\section{References}

Aksenova MV, Silvers JM, Aksenov MY, Nath A, Ray PD, Mactutus CF, Booze RM (2006) HIV-1 Tat neurotoxicity in primary cultures of rat midbrain fetal neurons: changes in dopamine transporter binding and immunoreactivity. Neurosci Lett 395:235-239.

Ambudkar IS, Ong HL (2007) Organization and function of TRPC channelosomes. Pflugers Arch 455:187-200.

Bachis A, Major EO, Mocchetti I (2003) Brain-derived neurotrophic factor inhibits human immunodeficiency virus-1/gp120-mediated cerebellar granule cell death by preventing gp120 internalization. J Neurosci 23:5715-5722.

Banisadr G, Gosselin RD, Mechighel P, Rostène W, Kitabgi P, Mélik Parsadaniantz S (2005) Constitutive neuronal expression of CCR2 chemokine receptor and its colocalization with neurotransmitters in normal rat brain: functional effect of MCP-1/CCL2 on calcium mobilization in primary cultured neurons. J Comp Neurol 492:178-192.

Bolin LM, Murray R, Lukacs NW, Strieter RM, Kunkel SL, Schall TJ, Bacon KB (1998) Primary sensory neurons migrate in response to the chemokine RANTES. J Neuroimmunol 81:49-57.

Bollimuntha S, Singh BB, Shavali S, Sharma SK, Ebadi M (2005) TRPC1mediated inhibition of 1-methyl-4-phenylpyridinium ion neurotoxicity in human SH-SY5Y neuroblastoma cells. J Biol Chem 280:2132-2140.

Bollimuntha S, Ebadi M, Singh BB (2006) TRPC1 protects human SH-SY5Y cells against salsolinol-induced cytotoxicity by inhibiting apoptosis. Brain Res 1099:141-149.

Chaudhuri P, Colles SM, Bhat M, Van Wagoner DR, Birnbaumer L, Graham LM (2008) Elucidation of a TRPC6-TRPC5 channel cascade that restricts endothelial cell movement. Mol Biol Cell 19:3203-3211.

Clapham DE (2003) TRP channels as cellular sensors. Nature 426:517-524.

Clapham DE, Runnels LW, Strübing C (2001) The TRP ion channel family. Nat Rev Neurosci 2:387-396.

De March Z, Giampà C, Patassini S, Bernardi G, Fusco FR (2006) Cellular localization of TRPC5 in the substantia nigra of rat. Neurosci Lett 402:35-39.

El Khoury J, Toft M, Hickman SE, Means TK, Terada K, Geula C, Luster AD (2007) Ccr2 deficiency impairs microglial accumulation and accelerates progression of Alzheimer-like disease. Nat Med 13:432-438.

Eugenin EA, D’Aversa TG, Lopez L, Calderon TM, Berman JW (2003) MCP-1 (CCL2) protects human neurons and astrocytes from NMDA or HIV-tat-induced apoptosis. J Neurochem 85:1299-1311.

Eugenin EA, Osiecki K, Lopez L, Goldstein H, Calderon TM, Berman JW (2006) CCL2/monocyte chemoattractant protein-1 mediates enhanced transmigration of human immunodeficiency virus (HIV)-infected leukocytes across the blood-brain barrier: a potential mechanism of HIV-CNS invasion and NeuroAIDS. J Neurosci 26:1098-1106.

Fujio Y, Walsh K (1999) Akt mediates cytoprotection of endothelial cells by vascular endothelial growth factor in an anchorage-dependent manner. J Biol Chem 274:16349-16354.

Glazner GW, Camandola S, Mattson MP (2000) Nuclear factor-kappaB mediates the cell survival-promoting action of activity-dependent neurotrophic factor peptide-9. J Neurochem 75:101-108.

Gosselin RD, Varela C, Banisadr G, Mechighel P, Rostene W, Kitabgi P, Melik-Parsadaniantz S (2005) Constitutive expression of CCR2 chemokine receptor and inhibition by MCP-1/CCL2 of GABA-induced currents in spinal cord neurones. J Neurochem 95:1023-1034.

Haughey NJ, Nath A, Mattson MP, Slevin JT, Geiger JD (2001) HIV-1 Tat through phosphorylation of NMDA receptors potentiates glutamate excitotoxicity. J Neurochem 78:457-467.

Heo JS, Han HJ (2006) ATP stimulates mouse embryonic stem cell proliferation via protein kinase $\mathrm{C}$, phosphatidylinositol 3-kinase/Akt, and mitogen-activated protein kinase signaling pathways. Stem Cells 24:2637-2648.

Itoh K, Mehraein P, Weis S (2000) Neuronal damage of the substantia nigra in HIV-1 infected brains. Acta Neuropathol 99:376-384.

Jia Y, Zhou J, Tai Y, Wang Y (2007) TRPC channels promote cerebellar granule neuron survival. Nat Neurosci 10:559-567.

Jiménez-Sainz MC, Fast B, Mayor F Jr, Aragay AM (2003) Signaling pathways for monocyte chemoattractant protein 1-mediated extracellular signal-regulated kinase activation. Mol Pharmacol 64:773-782.

Jung H, Toth PT, White FA, Miller RJ (2008) Monocyte chemoattractant protein-1 functions as a neuromodulator in dorsal root ganglia neurons. J Neurochem 104:254-263.

Kalkonde YV, Morgan WW, Sigala J, Maffi SK, Condello C, Kuziel W, Ahuja SS, Ahuja SK (2007) Chemokines in the MPTP model of Parkinson's disease: absence of CCL2 and its receptor CCR2 does not protect against striatal neurodegeneration. Brain Res 1128:1-11.

Kaul M, Ma Q, Medders KE, Desai MK, Lipton SA (2007) HIV-1 coreceptors CCR5 and CXCR4 both mediate neuronal cell death but CCR5 paradoxically can also contribute to protection. Cell Death Differ 14:296-305.

Kim SY, Bae JC, Kim JY, Lee HL, Lee KM, Kim DS, Cho HJ (2002) Activa- 
tion of p38 MAP kinase in the rat dorsal root ganglia and spinal cord following peripheral inflammation and nerve injury. Neuroreport 13:2483-2486.

Liu Y, Jones M, Hingtgen CM, Bu G, Laribee N, Tanzi RE, Moir RD, Nath A, He JJ (2000) Uptake of HIV-1 tat protein mediated by low-density lipoprotein receptor-related protein disrupts the neuronal metabolic balance of the receptor ligands. Nat Med 6:1380-1387.

Maggirwar SB, Sarmiere PD, Dewhurst S, Freeman RS (1998) Nerve growth factor-dependent activation of NF- $\kappa$ B contributes to survival of sympathetic neurons. J Neurosci 18:10356-10365.

Martorana A, Giampà C, DeMarch Z, Viscomi MT, Patassini S, Sancesario G, Bernardi G, Fusco FR (2006) Distribution of TRPC1 receptors in dendrites of rat substantia nigra: a confocal and electron microscopy study. Eur J Neurosci 24:732-738.

Meucci O, Fatatis A, Simen AA, Bushell TJ, Gray PW, Miller RJ (1998) Chemokines regulate hippocampal neuronal signaling and gp120 neurotoxicity. Proc Natl Acad Sci U S A 95:14500-14505.

Meucci O, Fatatis A, Simen AA, Miller RJ (2000) Expression of CX3CR1 chemokine receptors on neurons and their role in neuronal survival. Proc Natl Acad Sci U S A 97:8075-8080.

Montell C, Birnbaumer L, Flockerzi V (2002) The TRP channels, a remarkably functional family. Cell 108:595-598.

Paxinos G, Franklin KBJ (2001) The mouse brain in stereotaxic coordinates. San Diego: Academic.

Peng F, Dhillon N, Callen S, Yao H, Bokhari S, Zhu X, Baydoun HH, Buch S (2008) Platelet-derived growth factor protects neurons against gp120mediated toxicity. J Neurovirol 14:62-72.

Ramirez SH, Sanchez JF, Dimitri CA, Gelbard HA, Dewhurst S, Maggirwar SB (2001) Neurotrophins prevent HIV Tat-induced neuronal apoptosis via a nuclear factor-kappaB (NF-kappaB)-dependent mechanism. J Neurochem 78:874-889.

Ramsey IS, Delling M, Clapham DE (2006) An introduction to TRP channels. Annu Rev Physiol 68:619-647.

Ré DB, Przedborski S (2006) Fractalkine: moving from chemotaxis to neuroprotection. Nat Neurosci 9:859-861.

Rostène W, Kitabgi P, Parsadaniantz SM (2007) Chemokines: a new class of neuromodulator? Nat Rev Neurosci 8:895-903.

Singh IN, Goody RJ, Dean C, Ahmad NM, Lutz SE, Knapp PE, Nath A, Hauser KF (2004) Apoptotic death of striatal neurons induced by hu- man immunodeficiency virus-1 Tat and gp120: differential involvement of caspase-3 and endonuclease G. J Neurovirol 10:141-151.

Sodhi A, Biswas SK (2002) Monocyte chemoattractant protein-1-induced activation of p42/44 MAPK and c-Jun in murine peritoneal macrophages: a potential pathway for macrophage activation. J Interferon Cytokine Res 22:517-526.

Song L, Nath A, Geiger JD, Moore A, Hochman S (2003) Human immunodeficiency virus type 1 Tat protein directly activates neuronal $N$-methylD-aspartate receptors at an allosteric zinc-sensitive site. J Neurovirol 9:399-403.

Tarzami ST, Calderon TM, Deguzman A, Lopez L, Kitsis RN, Berman JW (2005) MCP-1/CCL2 protects cardiac myocytes from hypoxia-induced apoptosis by a $\mathrm{G}$ (alphai)-independent pathway. Biochem Biophys Res Commun 335:1008-1016.

Tong N, Perry SW, Zhang Q, James HJ, Guo H, Brooks A, Bal H, Kinnear SA, Fine S, Epstein LG, Dairaghi D, Schall TJ, Gendelman HE, Dewhurst S, Sharer LR, Gelbard HA (2000) Neuronal fractalkine expression in HIV-1 encephalitis: roles for macrophage recruitment and neuroprotection in the central nervous system. J Immunol 164:1333-1339.

Wang J, Shimoda LA, Sylvester JT (2004) Capacitative calcium entry and TRPC channel proteins are expressed in rat distal pulmonary arterial smooth muscle. Am J Physiol Lung Cell Mol Physiol 286:L848-L-858.

White FA, Sun J, Waters SM, Ma C, Ren D, Ripsch M, Steflik J, Cortright DN, Lamotte RH, Miller RJ (2005) Excitatory monocyte chemoattractant protein-1 signaling is up-regulated in sensory neurons after chronic compression of the dorsal root ganglion. Proc Natl Acad Sci USA 102:14092-14097.

Yao HH, Ding JH, Zhou F, Wang F, Hu LF, Sun T, Hu G (2005) Enhancement of glutamate uptake mediates the neuroprotection exerted by activating group II or III metabotropic glutamate receptors on astrocytes. J Neurochem 92:948-961.

Zauli G, Secchiero P, Rodella L, Gibellini D, Mirandola P, Mazzoni M, Milani D, Dowd DR, Capitani S, Vitale M (2000) HIV-1 Tat-mediated inhibition of the tyrosine hydroxylase gene expression in dopaminergic neuronal cells. J Biol Chem 275:4159-4165.

Zhou F, Yao HH, Wu JY, Yang YJ, Ding JH, Zhang J, Hu G (2006) Activation of group II/III metabotropic glutamate receptors attenuates LPSinduced astroglial neurotoxicity via promoting glutamate uptake. J Neurosci Res 84:268-277. 